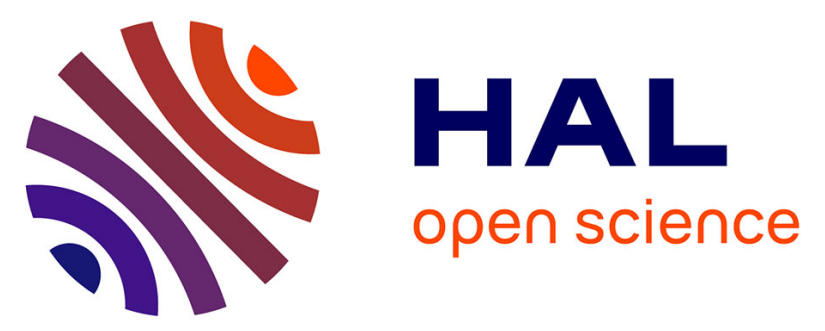

\title{
A New Technique for the Estimation of Cardiac Motion in Echocardiography Based on Transverse Oscillations: a preliminary evaluation in silico and a feasibility demonstration in vivo
}

Martino Alessandrini, Adrian Basarab, Loic Boussel, Xinxin Guo, André Serusclat, Denis Friboulet, Denis Kouamé, Olivier Bernard, Hervé Liebgott

\section{To cite this version:}

Martino Alessandrini, Adrian Basarab, Loic Boussel, Xinxin Guo, André Serusclat, et al.. A New Technique for the Estimation of Cardiac Motion in Echocardiography Based on Transverse Oscillations: a preliminary evaluation in silico and a feasibility demonstration in vivo. IEEE Transactions on Medical Imaging, 2014, vol. 33 ( $n^{\circ}$ 5), pp. 1148-1162. 10.1109/TMI.2014.2305846 . hal-01117833

\author{
HAL Id: hal-01117833 \\ https://hal.science/hal-01117833
}

Submitted on 18 Feb 2015

HAL is a multi-disciplinary open access archive for the deposit and dissemination of scientific research documents, whether they are published or not. The documents may come from teaching and research institutions in France or abroad, or from public or private research centers.
L'archive ouverte pluridisciplinaire $\mathbf{H A L}$, est destinée au dépôt et à la diffusion de documents scientifiques de niveau recherche, publiés ou non, émanant des établissements d'enseignement et de recherche français ou étrangers, des laboratoires publics ou privés. 


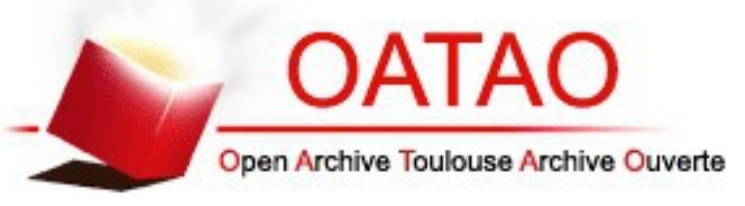

\section{Open Archive TOULOUSE Archive Ouverte (OATAO)}

OATAO is an open access repository that collects the work of Toulouse researchers and makes it freely available over the web where possible.

This is an author-deposited version published in : http://oatao.univ-toulouse.fr/ Eprints ID : 12909

To link to this article : DOI :10.1109/TMI.2014.2305846

URL : http://dx.doi.org/10.1109/TMI.2014.2305846

To cite this version : Alessandrini, Martino and Basarab, Adrian and Boussel, Loic and Guo, Xinxin and Serusclat, André and Friboulet, Denis and Kouamé, Denis and Bernard, Olivier and Liebgott, Hervé $A$ New Technique for the Estimation of Cardiac Motion in Echocardiography Based on Transverse Oscillations: a preliminary evaluation in silico and a feasibility demonstration in vivo. (2014) IEEE Transactions on Medical Imaging, vol. 33 ( $\left.{ }^{\circ} 5\right)$. pp. 1148-1162. ISSN 0278-0062

Any correspondance concerning this service should be sent to the repository administrator: staff-oatao@listes-diff.inp-toulouse.fr 


\title{
A New Technique for the Estimation of Cardiac Motion in Echocardiography Based on Transverse Oscillations: A Preliminary Evaluation In Silico and a Feasibility Demonstration In Vivo
}

\author{
Martino Alessandrini, Adrian Basarab, Loic Boussel, Xinxin Guo, André Serusclat, Denis Friboulet, \\ Denis Kouamé, Olivier Bernard, and Hervé Liebgott
}

\begin{abstract}
Quantification of regional myocardial motion and deformation from cardiac ultrasound is fostering considerable research efforts. Despite the tremendous improvements done in the field, all existing approaches still face a common limitation which is intrinsically connected with the formation of the ultrasound images. Specifically, the reduced lateral resolution and the absence of phase information in the lateral direction highly limit the accuracy in the computation of lateral displacements. In this context, this paper introduces a novel setup for the estimation of cardiac motion with ultrasound. The framework includes an unconventional beamforming technique and a dedicated motion estimation algorithm. The beamformer aims at introducing phase information in the lateral direction by producing transverse oscillations. The estimator directly exploits the phase information in the two directions by decomposing the image into two 2-D single-orthant analytic signals. An in silico evaluation of the proposed framework is presented on five ultra-realistic simulated echocardiographic sequences, where the proposed motion estimator is contrasted against other two phase-based solutions exploiting the presence of transverse oscillations and against block-matching on standard images. An implementation of the new beamforming strategy on a research ultrasound platform is also shown along with a preliminary in vivo evaluation on one healthy subject.
\end{abstract}

Index Terms-Cardiac strain, echocardiography, latreal displacements, motion estimation, multidimensional Hilbert transform, radio-frequency (RF) signal, transverse oscillations.

This work was supported by the French National Research Agency (ANR) under the US-Tagging Grant and partially supported by ANR-11LABX-0040-CIMI within the program ANR-11-IDEX-0002-02 of the University of Toulouse. It was performed within the framework of the LABEX PRIMES (ANR-11-LABX-0063) of Université de Lyon, within the program "Investissements d'Avenir" (ANR-11-IDEX-0007) operated by ANR. Asterisk indicates corresponding author.

M. Alessandrini, L. Boussel, X. Guo, D. Friboulet, O. Bernard, and H. Liebgott are with Université de Lyon, CREATIS; CNRS UMR5220; Inserm U1044; INSA-Lyon; Universit Lyon 1, F-69621 Villeurbanne Cedex, France (e-mail: liebgott@creatis.insa-lyon.fr).

A. Basarab and D. Kouamé are with Universit de Toulouse; Universit Paul Sabatier Toulouse 3; IRIT; CNRS UMR5505; F-31062 Toulouse Cedex 9, France (e-mail: adrian.basarab@irit.fr).

A. Serusclat is with the Department of Radiology, Louis Pradel Hospital, 69394 Lyon, France.

Digital Object Identifier 10.1109/TMI.2014.2305846

\section{INTRODUCTION}

C ARDIOVASCULAR diseases are the leading cause of deaths in the world $(48 \%)$, and it is projected that the annual number of deaths due to cardiovascular disease will increase from 17 millions in 2008 to 25 millions in 2030 [1]. In this context, clinical assessment of the cardiac function is essential for the diagnosis and treatment of heart diseases. Among available imaging techniques echocardiography has received special attention, since it offers high temporal resolution while being of relatively low cost. Moreover, cardiac motion estimation and the derived strain measures performed from ultrasound image sequences has proven to be a valuable tool for assessing cardiac function [2]-[6]. As a consequence, the development of motion estimation techniques from cardiac ultrasound data has a long history, dating back to the late eighties [7], and is still the topic of active research [8]-[12].

Following [13], most common approaches can be grouped in three main classes. A first family of methods is based on the differential technique known as optical flow. The earlier attempts towards automated cardiac motion estimation belong to this class [7], [14], [15]. Since they rely on the local analysis of spatial and temporal gradients, these methods may fail at estimating large inter-frame cardiac motion. This implies using multi-scale strategies or a block-matching initialization to provide a reliable first-order estimate of the displacement [16]-[18]. A second family is referred to as speckle tracking, and consists in finding the best match, as defined by the adopted similarity measure, between two blocks extracted from two subsequent frames. Most common similarity measures include cross-correlation (CC) [19], [20], sum of absolute differences (SAD) [21], or sum of squared differences (SSD) [22]. It was shown in [23] that these measures provide the maximum likelihood estimate of the displacement for a given statistical distribution of the image noise (Laplacian for SAD, Gaussian for SSD) and, following that observation, a new measure based on a Rayleigh distributed multiplicative noise was there introduced. Similar lines of reasoning have been exploited in [24]-[26]. Finally, several authors proposed to estimate cardiac motion by using nonrigid image registration, i.e., by computing a global deformation map warping a given frame on a reference one. The deformation field can be either discrete or 
parametric and is generally computed by minimizing a given cost function. In [27] the deformation field is represented on a B-spline basis and estimated by applying a SSD-like similarity measure to the image intensity. In [28] a discrete deformation function based on intensity and phase information is used. Since image registration is formulated as an inverse problem, it allows easily introducing a priori such as smoothness [11], [29], or incompressibility [8], [11].

Most of the approaches described above operate on conventional envelope-detected images, i.e., obtained through demodulation of the radio-frequency (RF) signal. Recently, several studies have proposed performing speckle tracking by using the RF signal instead. Since the RF signal contains high frequencies it is indeed better adapted to the estimation of small motions (typically on the order of the emitted pulse wavelength). This is done by using either time-domain correlation or phase difference estimation [30]. This type of technique is particularly used for cardiac elastography and examples include the work by Lubinski [31], Chen [32], D'hooge [33], Lopata [34], [35], and Konofagou [36]. RF-based speckle tracking is, however, currently not widespread in the field of echocardiography because its high motion sensitivity implies high frame rates [33], [37]. A comparison between envelope-detected and RF-based echocardiographic speckle tracking may be found in [34] and [38].

As noted in [34], any of the above-mentioned approaches faces an intrinsic limitation: the reduced lateral resolution (i.e., in the direction perpendicular to the beam propagation axis) and the absence of direct-phase information in the lateral direction results in a low accuracy in the computation of lateral displacements. Several algorithms have been described to tackle this issue, based on sophisticated interpolation schemes or re-correlation [4], [36], [39].

Another way around consists in modifying the image formation in order to introduce phase information in the lateral direction, i.e., by using a particular beam-forming step designed to produce transverse oscillations. This approach has been initiated by Jensen in the field of blood flow quantification [40], [41] and in ultrasound elastography [42], [43]. Preliminary results produced by our group recently extended this technique to echocardiography [44]-[47].

In this context, we describe in this paper a new setup for cardiac motion estimation, based on the following elements.

- A specific beamforming scheme for producing transverse oscillations (TO) in cardiac imaging, i.e., adapted to a sectorial acquisition geometry. As explained above, such approach allows introducing phase information in the lateral direction and thus improving accuracy of the 2-D motion estimation in this direction.

- A phase-based motion estimation algorithm specifically dedicated to the obtained TO images. This estimator locally constrains the motion to correspond to an affine transform and exploits the available 2-D phase of the TO images. Compared to previously published phase-based motion estimation methods (e.g., [45] or [46]), the approach presented herein combines the phases of two single-orthant analytical signals with an affine transformation instead of simple translations.
The accuracy of the proposed framework is evaluated in silico from five ultra-realistic simulated sequences [48] mimicking respectively three parasternal short-axis and two apical four chamber acquisitions. The new estimator is contrasted against other two phase-based estimators in [49] and [30] and conventional block-matching applied to standard images (i.e., without TO) [50]. Note that, although historically among the earlier techniques proposed for motion estimation in medical ultrasound [50], block-matching nonetheless still remains the methodology of choice [35], [51].

For each algorithm we evaluated the accuracy in recovering the simulated displacement field and in computing the cardiac strain. All algorithms exploiting transverse oscillations were found to estimate more accurately the lateral component of the displacement than standard block matching and this led to an overall better precision in the computation of the total displacement field and of the cardiac strain. Among the phase-based techniques considered the proposed algorithms was found to be in average the more accurate and reliable. An implementation of the new beamforming technique on an ultrasound research platform [52] is also presented along with a preliminary in vivo evaluation of the proposed motion estimation framework for the computation of cardiac strain on one healthy subject. Computed strain curves were in line with what reported in literature for an healthy heart.

The paper is structured as follows. Section II describes the generation of the transverse oscillations for sectorial cardiac acquisition. Section III presents the motion estimation algorithm. Section IV describes the numerical experiments used to evaluate the proposed framework and provides the download link for an implementation of the presented motion estimator. Section V presents the obtained results, which are discussed in Section VI.

\section{TRANSVERSE OSCILLATION ULTRASOUND IMAGES}

Transverse oscillation (TO) ultrasound images exhibit in the lateral direction the same kind of oscillations normally present in the axial direction [41]. Lateral oscillations are obtained by implementing a point spread function (PSF) presenting, in addition to the common axial modulation, a modulation in the lateral direction. TO modality was originally developed for linear probes where, denoting by $x$ and $z$ lateral and axial coordinates respectively, the system PSF can be written as [53] $h(x, z)=$ $h_{x}(x) h_{z}(z)$ with

$$
h_{\xi}(\xi)=e^{-\pi \frac{\xi^{2}}{\sigma_{\xi}^{2}}} \cos \left(2 \pi \frac{\xi}{\lambda_{\xi}}\right)
$$

where $\xi=\{x, z\}, \lambda_{x}\left(\lambda_{z}\right)$ is the lateral (axial) oscillation wavelength and $\sigma_{x}\left(\sigma_{z}\right)$ is the lateral (axial) full-width half-maximum (FWHM) of the Gaussian envelope [42].

The axial profile of the PSF is related to the excitation pulse and the impulse response of the transducer elements used. As a consequence, the axial modulation is naturally present in the axial PSF profile and the weighting window can be adapted using specific excitation pulses. The degrees of freedom that enable one to control the transverse profile of the PSF are instead the delay between the elements used in transmit and in 
receive and the weighting coefficients applied to each element in transmit and in receive.

To design these parameters the Fraunhofer approximation is commonly used [54]. Fraunhofer approximation applies to focused acoustic beams and states that, at the focal point, the lateral beam profile $h_{x}(x)$ and the radiating aperture $w(x)$ are related by the Fourier transform [54]

$$
h_{x}\left(x_{0}\right) \propto \int_{\text {Aperture }} w(x) e^{-j \frac{2 \pi}{\lambda_{z} z} x_{0} x} d x .
$$

From (2) it is straightforward to show that a bi-modal apodization function of the kind [42]

$$
w(x) \propto e^{-\pi\left(\frac{x-x_{0}}{\sigma_{0}}\right)^{2}}-e^{-\pi\left(\frac{x+x_{0}}{\sigma_{0}}\right)^{2}}
$$

realizes the desired lateral profile, with $x_{0}=z \lambda_{z} / \lambda_{x}$ and $\sigma_{0}=$ $\sqrt{2} \lambda_{z} z / \sigma_{x}$. As these parameters depend on the axial coordinate, the apodization function has to be dynamically adjusted in order to obtain a depth-invariant PSF [53].

\section{A. Transverse Oscillations in Echocardiography}

To date most consolidated applications of TO are blood flow imaging [40], [41], [55] and elastography [42], [43]. In both cases the presence of lateral oscillations has been shown to favor a more accurate estimation of lateral displacements as compared to traditional beamforming techniques.

Recently, the concept of TO has been extended to cardiac ultrasound, where the accurate quantification of lateral heart deformations still remains a challenge [56]. Our group had a pioneering role in studying the feasibility of TO imaging in echocardiography [44]-[47]. In particular, a beamforming technique was presented in [44] for the generation of TO on sectorial probes of common use in cardiac applications. The beamformer design relies on the principle of back-propagation and allows to obtain on pre-scan converted data (i.e., in polar coordinates) the same kind of lateral oscillations otherwise possible on linear probes. More specifically, a PSF completely analogous to the one in (1) can be obtained in the polar space $(\rho, \theta)$. This is done according to the coordinate transformation $z \approx \rho$ and $x \approx \rho \theta$ and, consequently, the parameters transformation $\lambda_{x} \approx \lambda_{\theta} \rho$, $\sigma_{x} \approx \sigma_{\theta} \rho, \lambda_{z} \approx \lambda_{\rho}$ and $\sigma_{z} \approx \sigma_{\rho}$. The required apodization function has the same form as (3), where the peaks position and width are given by $x_{\theta_{0}}=\lambda_{\rho} / \lambda_{\theta}$ and $\sigma_{\theta_{0}}=\sqrt{2} \lambda_{\rho} / \sigma_{\theta}$. Interestingly, these quantities are no longer depth-dependent as in linear geometries. As a result a space invariant PSF can be obtained on sector scan without dynamically modifying the apodization function. For more detail we address the reader to [44].

\section{MOtION ESTIMATION AlgORITHM FOR ECHOCARDIOGRAPHIC IMAGES WITH TRANSVERSE OSCILLATIONS}

Consider two rectangular blocks of pixels extracted from two subsequent RF frames $s\left(x, z, t_{0}\right)$ and $s\left(x, z, t_{1}\right)$ (for simplicity $\left.t_{1}=t_{0}+1\right)$ of a cardiac ultrasound sequence. The motion estimation problem consists in computing the displacement field $\mathbf{d}(x, z)=\left[d_{1}(x, z), d_{2}(x, z)\right]^{T}$ mapping the second block onto the first, being $d_{1}$ and $d_{2}$ the lateral and axial components of the displacement respectively. This is normally done by adopting the so-called brightness constancy assumption $s\left(x, z, t_{0}\right)=s\left(x-d_{1}(x, z), z-d_{2}(x, z), t_{1}\right)$.

Nevertheless, it has been shown that brightness conservation can be a misleading assumption as far as cardiac ultrasound images are concerned [18]. The reason is that the amplitude of the backscattered echo depends on the angle formed by the acoustic beam and the myocardial fibers, which obviously varies in time due to the heart motion. As a consequence, the same portion of tissue will return different echoes after it position has changed. For this reason, we replace the classical brightness constancy assumption with a more robust phase constancy assumption. Image phase is indeed ideally suited for ultrasound images since it is independent on the local intensity while intrinsically related to the local image structure.

In particular, 2-D single-orthant analytic signals are used to compute the image phase [57]. Based on multidimensional Hilbert transforms, they represent one of the first attempts to generalize the classical 1-D analytic signal to $n$ dimensions (as for example for 2-D images). The suitability of single-orthant analytic signals for modeling and processing TO ultrasound images has been shown in different contexts from the considered one in [49], [58].

Based on the TO theory presented in the previous section, a signal model consisting of a 2-D spatial modulation at spatial frequencies $1 / \lambda_{x}$ and respectively $1 / \lambda_{z}$ can be assumed [49]

$$
s\left(x, z, t_{0}\right)=w_{s}\left(x, z, t_{0}\right) \cos \left(2 \pi x / \lambda_{x}\right) \cos \left(2 \pi z / \lambda_{z}\right)
$$

where $w_{s}$ is a low-pass 2-D window having its highest frequency lower than the frequency of the 2-D cosinus (a reasonable hypothesis in TO ultrasound imaging).

The four single-orthant analytic signals are then calculated by canceling three of the four quadrants in the 2-D spectrum. However, given the symmetry of the 2-D Fourier transform of real images, these analytic signals contain, two by two, redundant information [49]. For this reason, we only conserve two of the four available single-orthant analytic signals. Following [49], they can be expressed in the frequency domain as

$$
\begin{aligned}
& S_{s o 1}\left(f_{1}, f_{2}, t\right)=S\left(f_{1}, f_{2}, t\right)\left(1+\operatorname{sign}\left(f_{1}\right)\right)\left(1+\operatorname{sign}\left(f_{2}\right)\right) \\
& S_{s o 2}\left(f_{1}, f_{2}, t\right)=S\left(f_{1}, f_{2}, t\right)\left(1-\operatorname{sign}\left(f_{1}\right)\right)\left(1+\operatorname{sign}\left(f_{2}\right)\right)
\end{aligned}
$$

where capitals letters denote the 2-D Fourier transform, $f_{1}$ and $f_{2}$ denote the lateral and axial frequency respectively and $\operatorname{sign}(x)=x /|x|$. By denoting $\Phi_{s o 1}(x, z, t)$ and $\Phi_{s o 2}(x, z, t)$ the spatial phases associated to the two considered analytic signals, the phase constancy assumption reads as

$$
\begin{aligned}
& \left(\begin{array}{l}
\Phi_{s o 1}\left(x, z, t_{1}\right) \\
\Phi_{s o 2}\left(x, z, t_{1}\right)
\end{array}\right) \\
& =\left(\begin{array}{l}
\Phi_{s o 1}\left(x+d_{1}(x, z), z+d_{2}(x, z), t_{0}\right) \\
\Phi_{s o 2}\left(x+d_{1}(x, z), z+d_{2}(x, z), t_{0}\right)
\end{array}\right)
\end{aligned}
$$

Assuming small displacements, as commonly done in differential techniques, the right-hand side of (6) can be replaced by 
its first-order Taylor development and this leads to the linear system of equations [49]

$$
\left(\begin{array}{c}
\Phi_{s o 1}^{(t)}(x, z) \\
\Phi_{s o 2}^{(t)}(x, z)
\end{array}\right)=\mathbf{J} \cdot\left(\begin{array}{c}
d_{1}(x, z) \\
d_{2}(x, z)
\end{array}\right)
$$

where $\Phi_{s o 2}^{(t)}(x, z)$ and $\Phi_{s o 2}^{(t)}(x, z)$ are the temporal derivatives of $\Phi_{s o 1}$ and $\Phi_{s o 2}$ respectively while

$$
\mathbf{J}(x, z, t)=2 \pi\left(\begin{array}{cc}
1 / \lambda_{x} & 1 / \lambda_{z} \\
-1 / \lambda_{x} & 1 / \lambda_{z}
\end{array}\right)
$$

is the Jacobian matrix of the vector $\left[\Phi_{s o 1}, \Phi_{s o 2}\right]^{T}$.

While the motion estimation problem (7) could be in principle solved pixel-wise, the corresponding solution would be highly sensitive to noise, which is not acceptable in a low SNR context as medical ultrasound. The common way around this is to solve the problem in the least squares sense by assuming that all the pixels in a block translate of the same quantity, i.e., $\mathbf{d}(x, z)=\mathbf{d}_{0}$. Nevertheless, several studies pointed out that the simple translation model is too restrictive in the context of cardiac motion estimation [17], [18]. In this scenario a much better solution is instead represented by the affine model [17], [18]. Considering for simplicity a block centered at $\left(x_{0}, z_{0}\right)=(0,0)$, the affine model is

$$
\mathbf{d}(x, z)=\mathbf{A}(x, z) \mathbf{u}, \quad \mathbf{A}=\left[\begin{array}{llllll}
1 & 0 & x & z & 0 & 0 \\
0 & 1 & 0 & 0 & x & z
\end{array}\right]
$$

where $\mathbf{u}=\left[d_{10}, d_{20}, d_{1 x}, d_{1 z}, d_{2 x}, d_{2 z}\right]^{T}$ is the new unknown vector: $d_{10}$ and $d_{20}$ correspond to the translation of the window center and $d_{i k}=\partial_{k} d_{i}$.

By plugging (9) into (7) and after suitable rearrangement of the system entries, it can be shown (see Appendix A) that the motion estimation problem can be independently solved for the two main directions $x$ and $z$ as

$$
\begin{aligned}
& \frac{\lambda_{x}}{4 \pi}\left(\begin{array}{c}
\Phi_{s o 1}^{(t)}\left(x_{0}, z_{0}\right)-\Phi_{s o 2}^{(t)}\left(x_{0}, z_{0}\right) \\
\cdots \\
\Phi_{s o 1}^{(t)}\left(x_{N-1}, z_{N-1}\right)-\Phi_{s o 2}^{(t)}\left(x_{N-1}, z_{N-1}\right)
\end{array}\right) \\
& =\left(\begin{array}{ccc}
1 & x_{0} & z_{0} \\
\cdots & \cdots & \cdots \\
1 & x_{N-1} & z_{N-1}
\end{array}\right)\left(\begin{array}{l}
d_{10} \\
d_{1 x} \\
d_{1 z}
\end{array}\right)
\end{aligned}
$$

and

$$
\begin{aligned}
& \frac{\lambda_{z}}{4 \pi}\left(\begin{array}{c}
\Phi_{s o 1}^{(t)}\left(x_{0}, z_{0}\right)+\Phi_{s o 2}^{(t)}\left(x_{0}, z_{0}\right) \\
\cdots \\
\Phi_{s o 1}^{(t)}\left(x_{N-1}, z_{N-1}\right)+\Phi_{s o 2}^{(t)}\left(x_{N-1}, z_{N-1}\right)
\end{array}\right) \\
& =\left(\begin{array}{ccc}
1 & x_{0} & z_{0} \\
\cdots & \cdots & \cdots \\
1 & x_{N-1} & z_{N-1}
\end{array}\right)\left(\begin{array}{c}
d_{20} \\
d_{2 x} \\
d_{2 z}
\end{array}\right)
\end{aligned}
$$

where $\left(x_{k}, z_{k}\right)(k=0,1, \ldots, N-1)$ denotes the coordinate of the $k$ th pixel of the considered block.

The two overdetermined systems (10) and (11) are then solved by classical least-squares fitting. We also remember that given two complex numbers the sum of their phases is equal to the phase of their product while the difference of their phases is equal to the phase of the product of the first with the conjugate of the second. These relations are better used in the motion estimation framework to compute phase sums and differences, since they allow avoiding tedious unwrapping issues.

We also note that, since the phase of (4) does not change for horizontal (vertical) shifts equal to the wavelength $\lambda_{x}\left(\lambda_{z}\right)$, then the largest displacements that can be estimated unambiguously in the lateral and axial direction respectively are limited to $\lambda_{x} / 2$ and $\lambda_{z} / 2$.

Finally, while the motion estimation algorithm has been presented for Cartesian coordinates $(x, z)$, as said in Section II, exactly the same considerations apply to pre-scan converted sectorial data simply by replacing $(x, z)$ by the polar couple $(\rho, \theta)$.

\section{Materials And Methods}

\section{A. Evaluation Data Set}

A quantitative performance evaluation of the proposed framework was made in silico. A preliminary feasibility study in vivo will be shown in the results section.

For the in silico evaluation we made use of ultra-realistic synthetic echocardiographic image sequences generated according to an original framework we recently developed [48]. Briefly, cardiac motion is mimicked by displacing a set of point scatterers over time. Both scatter amplitude and motion are learned from a real echocardiographic acquisition adopted as a template. From the time-variant scatter map Field II is employed to simulate the image formation process [59], [60]. Since the synthetic cardiac motion is known, this can be used to benchmark motion estimation algorithms.

The resulting synthetic sequences are extremely realistic both in their motion and aspect, to the point it is hard to distinguish them from real clinical recordings. In particular, all the typical image artifacts such as reverberations, clutter noise, signal dropout, local intensity and/or contrast variations over time due to changing cardiac fiber orientation, which have a major impact on the performance of algorithms for motion/deformation estimation, are naturally present as they are inherited from the template sequence [48]. As a consequence, although obtained on synthetic data, the reported evaluation is well representative of what could be expected on real clinical data.

Five synthetic sequences were generated according to [48], namely three parasternal short-axis (SAx) and two apical four chamber (A4C), which correspond to two of the most commonly employed views in the clinical practice [61]. In the following the three SAx sequences will be denoted as SAx1, SAx2, and SAx3 while the two A4C sequences will be denoted as $\mathrm{A} 4 \mathrm{C} 1$ and $\mathrm{A} 4 \mathrm{C} 2$. For each of the five sequences the two acquisition modalities of interest were simulated, i.e., traditional beamforming and TO. Note that the time variant scatter map associated to a synthetic sequence, and hence the benchmark motion field, remained unchanged when modifying the beamforming strategy.

The two acquisition strategies were implemented by suitably setting the receive apodization function of the synthetic probe used by Field II: a standard Hanning window was used in the standard case while the bimodal function in (3) was used in the case with TO. In this latter case the parameters $\lambda_{\theta}=6^{\circ}$, $\sigma_{\theta}=4 \lambda_{\theta}$ were used, while the remaining probe settings were 


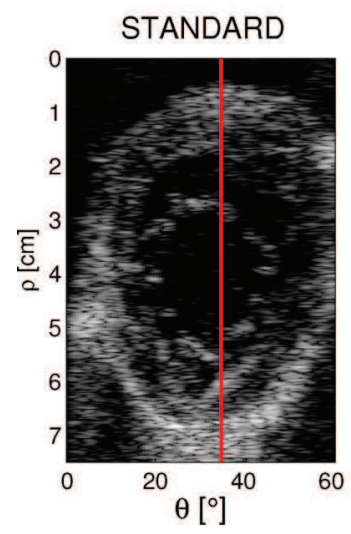

(a)

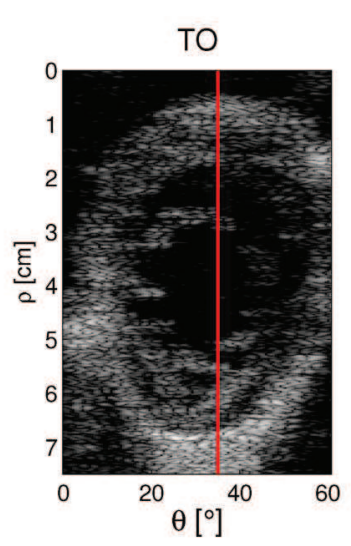

(b)

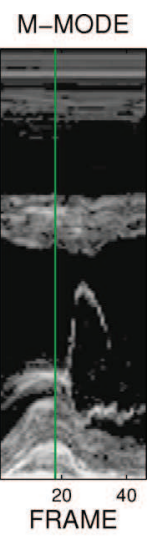

(c)
Fig. 1. Comparison of standard beamforming and transverse oscillations: (a) and (b) compare the same frame of the SAx3 sequence in the two acquisition modalities while (c) illustrates the M-mode computed over one cardiac cycle on the scan-line represented by the red segment. End systole (ES) has been assigned in correspondence of the highest muscle contraction and is denoted by a dark green line. For the SAx3 sequence ES corresponds roughly to frame 18 .

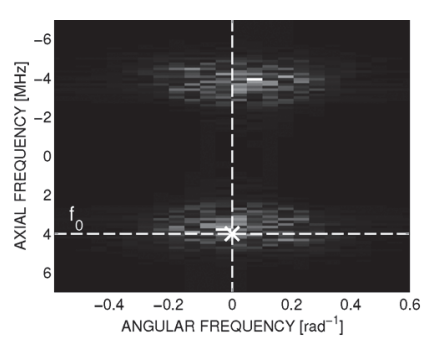

(a)

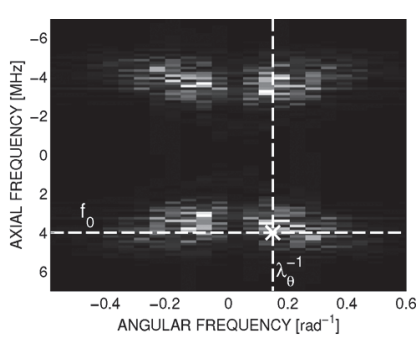

(b)
Fig. 2. Amplitude spectrum of the RF images corresponding to the two SAx frames in Fig. 1(a) and (b).

kept constant for both acquisition modalities, namely center frequency $f_{0}=4 \mathrm{MHz}$, sampling frequency $f_{s}=40 \mathrm{MHz}$, speed of sound $c=1540 \mathrm{~m} / \mathrm{s}$ and 64 elements. Sweep angle were $65^{\circ}$ for the SAx sequences and $75^{\circ}$ for the A4C sequences. Frame rate was 50 frames/s for the three SAx sequences and 45 frames/s for the two A4C ones.

Examples of simulated images are given in Fig. 1. Since the TO model (1) holds for pre-scan converted data, images are reported in the polar domain $(\rho, \theta)$. It is evident how the presence of lateral oscillations reflects in a richer speckle pattern as compared to traditional beamforming. Fig. 1 only allows assessing the visual realism of the individual frames. In order to appreciate the dynamical behavior the reader is addressed to the videos posted online. ${ }^{1}$

The Fourier spectrum of two simulated frames (radio frequency images are considered for the frequency analysis) obtained with standard beamforming and TO is reported in Fig. 2(a) and (b), respectively. Note the effect of the lateral modulation at the angular frequency $1 / \lambda_{\theta}$ in the case with TO.

\section{B. Cardiac Motion Estimation}

As reference technique for standard RF images we will consider block matching (BM). On RF images with TO the estimator

${ }^{1}$ Available online: http://www.creatis.insa-lyon.fr/us-tagging/node/13 described in Section III, referred to as Affine Phase Based Estimator (APBE), will be contrasted against other two phase-based solutions: a previous version of the same estimator [49], referred to as Translation Phase Based Estimator (TPBE), where a simple translation model is considered instead of the affine model in (9), and a technique based on maximizing block-wise the correlation between phase images, referred to as phase correlation estimator (PhCorr). The latter, based on an iterative Newton algorithm, estimates the displacement by searching for the phase root of the complex cross-correlation function [30]. For one block of pixels, PhCorr method was implemented to estimate the displacements of all the columns and rows, and the final estimates were the mean values for each direction.

Considering RF data prevents axial down-sampling and this implies dealing with axial displacements which, at conventional sampling rates, are easily of the order of few tens of pixels. As an example, at the considered sampling rate $\left(f_{s}=40 \mathrm{MHz}\right)$ and speed of sound ( $c=1540 \mathrm{~m} / \mathrm{s})$, a displacement of $1 \mathrm{~mm}$ in the direction of the beam propagation would correspond to a shift of $\sim 52$ pixels.

Displacements of such entity violate the small displacements assumption essential in differential techniques as the proposed one. We dealt with this issue by proceeding in two steps: an initialization phase to produce a coarse estimate of the displacement and a successive refinement, where the proposed phase based estimator was applied to estimate the residual motion.

The initialization was performed with block-matching with sums of absolute differences as similarity criterion. At this stage no interpolation (i.e., no sub-pixel precision) was used in order to speedup the procedure. Initialization was not performed on the RF directly but on the B-mode. Indeed B-mode images, being base-band, are better suited for the analysis of large deformations than RF [38].

In order to have a fair comparison among the four considered algorithms, the same initialization was kept both when the refinement was made on standard RF (the case of BM) and on TO RF (the case of the APBE and TBPE algorithms). In particular the initial estimate was obtained from B-mode images without transverse oscillations.

The initialization procedure is summarized in Fig. 3. The block-matching initialization $\mathbf{D}=\left[D_{1}, D_{2}\right]$ determines the two blocks $s_{1}$ and $s_{2}$ for the successive refinement $\Delta \mathrm{D}$. The total displacement is then given by $\mathbf{D}+\Delta \mathbf{D}$. For what concerns the algorithm in Section III, the fact that the two blocks are not aligned only implies replacing in (10) and (11) $\Phi_{s o 2}\left(x_{i}, z_{i}\right)$ with $\Phi_{s o 2}\left(x_{i}+D_{1}, z_{i}+D_{2}\right)$. The parameters $\left[L_{1}, L_{2}\right]$ and $\left[G_{1}, G_{2}\right]$ defined in Fig. 3 were the only required. They were optimized in order to have the smallest estimation error (see next section for more details) on the synthetic SAx3 sequence. The optimal found values (in pixels) corresponded to: $L_{1}=16, L_{2}=64$, $G_{1}=3$ and $G_{2}=64$. The RF image size was $4562 \times 113$ pixels $^{2}$.

When block-matching was employed for the refinement interpolation factors of 1 and 6 were used in the axial and lateral directions respectively. Note that, due to the high sampling frequency of RF data, an interpolation of 1 in the axial direction was sufficient to obtain a resolution of $c /\left(2 f_{s}\right) \approx 0.02 \mathrm{~mm}$. 


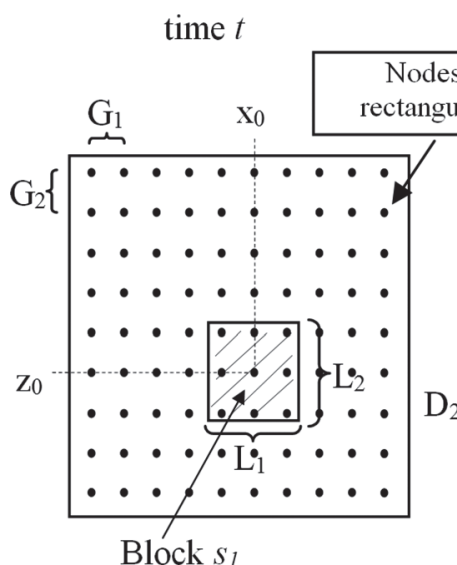

(a) time $t+1$

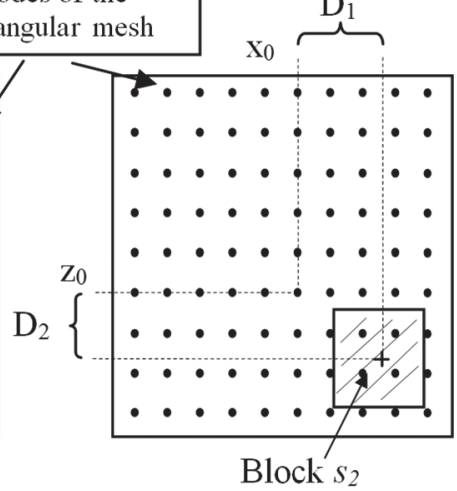

(b)
Fig. 3. Initialization procedure. (a) Frame at time $t$. (b) Frame at time $t+$ 1. Centers of the considered blocks are represented as black dots. Block $s_{1}$ is centered at $\left(x_{0}, z_{0}\right) . D_{1}$ and $D_{2}$ are the lateral and axial initial estimates given by block-matching and defining the center of the block $s_{2} . G_{1}$ and $G_{2}$ define the spacing between the nodes. $L_{1}$ and $L_{2}$ define the block size. Successive refinement will be on $s_{1}$ and $s_{2}$

Let us finally point out that, since the TO image model holds only for pre-scan converted data, in the proposed framework motion estimation must be performed in polar coordinates. The computed displacement field has then to be scan converted to have the values in Cartesian coordinates.

The MATLAB implementation of the APBE algorithm is made freely available for downloaded online. ${ }^{2}$

\section{Accuracy Assessment}

The four algorithms were compared in terms of accuracy in the recovered displacement field and in the computed cardiac strain.

1) Displacement Field: Let us denote as $\overline{\mathbf{d}}_{k}(x, z)=$ $\left[\bar{d}_{k, 1}(x, z), \bar{d}_{k, 2}(x, z)\right]$ the ground-truth displacement between frame $k$ and frame $k+1$ at position $(x, z)$ and as $\mathbf{d}_{k}(x, z)=\left[d_{k, 1}(x, z), d_{k, 2}(x, z)\right]$ the estimated one.

The results in polar coordinates were considered first. Hereto the main goal is to show the improvement in the estimation of lateral displacements made possible thanks to the proposed framework. Lateral and axial errors were used for this purpose

$$
\begin{aligned}
& \operatorname{err}_{k, \text { lat }}(x, z)=\left|\bar{d}_{k, 1}(x, z)-d_{k, 1}(x, z)\right| \\
& \operatorname{err}_{k, \text { ax }}(x, z)=\left|\bar{d}_{k, 2}(x, z)-d_{k, 2}(x, z)\right|
\end{aligned}
$$

where $|\cdot|$ denotes the absolute value. Error study was limited to the region of the left-ventricle muscle. The latter was manually contoured from the first frame of each synthetic sequence. The mask was then propagated to all the frames of the sequence by using the benchmark motion field. The value of these error metrics will be reported in pixels.

Cardiac ultrasound sequences are commonly visualized and processed in Cartesian coordinates, i.e., after scan conversion of the beamformed images. For this reason the remaining part of the evaluation considered scan converted images. In this case,

\footnotetext{
${ }^{2}$ Available online: http://www.creatis.insa-lyon.fr/us-tagging/node/13
}

the accuracy in the recovered displacement was measured with the endpoint error [62]

$$
E E_{k}(x, z)=\left\|\overline{\mathbf{d}}_{k}^{\mathrm{SC}}(x, z)-\mathbf{d}_{k}^{\mathrm{SC}}(x, z)\right\|_{2}
$$

where $\overline{\mathbf{d}}_{k}^{\mathrm{SC}}(x, z)$ and $\mathbf{d}_{k}^{\mathrm{SC}}(x, z)$ denote the reference and the estimated displacement after scan conversion and $\|\cdot\|_{2}$ is the $\ell_{2}$-norm. Errors in Cartesian coordinates were measured in millimeters.

2) Strain: Cardiac strain was measured similarly to [63]. The endocardium was first manually contoured in the ED frame (i.e., the first frame of the sequence). A ROI for strain estimation was then created by expanding the endocardial contour along its normal to represent the myocardium. This region was subsequently populated in the directions normal and tangential to the endocardial contour with 6 and 100 sample points respectively, and given a label corresponding to one of the heart segments. Segments were established following the guidelines given by the American Heart Associated (AHA) [64]. Namely, six equally spaced segments around the circumference were considered for $\mathrm{SaX}$ views while, for what concerns apical views, three equally spaced longitudinal levels were defined from base to apex, either on the septal or lateral side, thus leading again to six segments.

The test points were then displaced over the full cardiac cycle by using the reference displacement and the displacement estimated by each algorithm. The strain along a direction $\mathbf{n}$ at time $k$ was then computed as [29], [63]

$$
\epsilon_{\mathbf{n}}(k)=\frac{D_{\mathbf{n}}(k)}{D_{\mathbf{n}}(0)}-1
$$

where $D_{\mathbf{n}}(k)$ denotes the distance between two consecutive test points. More precisely, normal and tangential directions on $\mathrm{SaX}$ sequences were used to determine radial and circumferential strain components $\left(\epsilon_{R R}\right.$ and $\left.\epsilon_{C C}\right)$ respectively, while the tangential directions on apical sequences was used to determine the longitudinal component $\epsilon_{L L}$.

Note that each simulated sequence corresponds to one full cycle from one end-diastole to the following. Given the periodicity of the cardiac cycle it is therefore reasonable to assume $\epsilon_{\mathbf{n}}\left(N_{F}\right)=0$ being $N_{F}$ the number of frames in the sequence. As in [29], [63], this condition is imposed by applying the following drift compensation to the computed strain curves. The strain compensated strain $\epsilon^{d c}$ is

$$
\epsilon_{\mathbf{n}}^{d c}(k)=\epsilon_{\mathbf{n}}(k)-\frac{k-1}{N_{F}-1} \epsilon_{\mathbf{n}}\left(N_{F}\right) .
$$

Segmental strain was obtained by averaging the strain values computed point-wise on the test points on each segment.

3) Statistical Analysis: For what concerns the accuracy in retrieving the displacement field, the statistical significance of the differences among the four algorithms was tested by means of the Friedman rank test $(\alpha=0.05)$ in conjunction with the post-hoc test proposed by Daniel [65], as suggested in [66]. Strain accuracy was instead assessed by using the Pearson correlation coefficient $\rho$ together with the bias $\mu$ and standard deviation $\sigma$ returned by the Bland-Altman (BA) analysis. For each 
TABLE I

Horizontal Error (Mean Value \pm Standard Deviation). VAlues in PiXels

\begin{tabular}{lccccc} 
& SAx1 & SAx2 & SAx3 & A4C1 & A4C2 \\
\hline APBE & $0.153 \pm 0.139$ & $0.131 \pm 0.119$ & $0.192 \pm 0.186$ & $0.307 \pm 0.292$ & $0.202 \pm 0.174$ \\
TPBE & $0.170 \pm 0.167$ & $0.147 \pm 0.147$ & $0.233 \pm 0.232$ & $0.365 \pm 0.369$ & $0.214 \pm 0.192$ \\
BM & $0.180 \pm 0.216$ & $0.206 \pm 0.237$ & $0.331 \pm 0.365$ & $0.440 \pm 0.430$ & $0.256 \pm 0.290$ \\
PhCorr & $0.148 \pm 0.163$ & $0.135 \pm 0.151$ & $0.208 \pm 0.229$ & $0.455 \pm 0.447$ & $0.204 \pm 0.213$ \\
\hline
\end{tabular}

TABLE II

Vertical Error (Mean Value \pm Standard Deviation). Values in Pixels

\begin{tabular}{lccccc} 
& SAx1 & SAx2 & SAx3 & A4C1 & A4C2 \\
\hline APBE & $1.0478 \pm 2.2246$ & $1.0114 \pm 1.6374$ & $2.0473 \pm 3.4013$ & $1.9055 \pm 3.8254$ & $0.5657 \pm 1.7386$ \\
TPBE & $0.8705 \pm 2.3213$ & $0.8669 \pm 1.7177$ & $1.8538 \pm 3.5787$ & $1.7467 \pm 4.1863$ & $0.4829 \pm 1.9180$ \\
BM & $0.9672 \pm 2.3697$ & $0.9150 \pm 1.7659$ & $1.8861 \pm 3.6915$ & $1.4724 \pm 4.2151$ & $0.5519 \pm 1.9180$ \\
PhCorr & $0.9027 \pm 2.3535$ & $0.8508 \pm 1.7498$ & $1.9344 \pm 3.6270$ & $5.9907 \pm 7.3476$ & $0.5795 \pm 1.9456$ \\
\hline
\end{tabular}

TABLE III

Endpoint Error (Mean Value \pm Standard Deviation) After Scan Conversion. Values in Millimeters

\begin{tabular}{lccccc} 
& SAx1 & SAx2 & SAx3 & A4C1 & A4C2 \\
\hline APBE & $0.104 \pm 0.112$ & $0.095 \pm 0.092$ & $0.137 \pm 0.146$ & $0.119 \pm 0.124$ & $0.084 \pm 0.091$ \\
TPBE & $0.115 \pm 0.133$ & $0.105 \pm 0.115$ & $0.162 \pm 0.178$ & $0.133 \pm 0.144$ & $0.089 \pm 0.103$ \\
BM & $0.130 \pm 0.177$ & $0.151 \pm 0.192$ & $0.231 \pm 0.279$ & $0.174 \pm 0.207$ & $0.112 \pm 0.167$ \\
PhCorr & $0.105 \pm 0.135$ & $0.101 \pm 0.124$ & $0.150 \pm 0.178$ & $0.212 \pm 0.220$ & $0.085 \pm 0.109$ \\
\hline
\end{tabular}

correlation value the $\mathrm{p}$-value was computed testing the hypothesis of no correlation. The statistical significance of each reported bias $\mu$ was measured with a t-test. Fisher's z-transform ( $\alpha=0.05)$ was used to compare the strengths of different correlations. T-test $(\alpha=0.05)$ was used to compare the biases returned by the BA analysis.

Segmental strain values were considered and all segments were included in the analysis. The three strain components $\epsilon_{R R}, \epsilon_{C C}$ and $\epsilon_{L L}$ were considered independently. Among the different phases in the cardiac cycle, the strain at end systole has been shown to be particularly relevant for diagnosis [12]. Hereto the accuracy in computing end-systolic strain values was measured separately and will be presented in the results section. Nevertheless, considering a single time instant reduced the sample size to a point that statistical significance was never observed. In order to have more statistically significant results, and together to have a more exhaustive look at the strain behavior over time, the analysis was repeated by including multiple time instants obtained by sampling one frame out of eight. Temporal sub-sampling was adopted to avoid correlation.

Let us finally note that no post processing operation such as low-pass or median filtering was performed on the estimated displacement fields. This was done to have a direct understanding of the relation between the data type (standard beamforming and TO) and the accuracy possible in the computation of displacements.

\section{RESULTS}

\section{A. In Silico Results}

1) Accuracy in the Displacement Field: Tables I and II report respectively the lateral and axial displacement errors measured in pixels on the five simulated sequences before scan conversion while Table III reports the errors measured in millimeters after scan conversion. Mean values and standard deviations are computed for each sequence by including all pixel estimates and all frames. An analysis of the errors behavior over time is provided by Fig. 4.

From Table I all algorithms exploiting TO images almost consistently return in average more accurate lateral motion estimates than regular block matching on standard RF images, with the only exception of sequence $\mathrm{A} 4 \mathrm{C} 1$ where the PhCorr algorithm performs the worst. This result reveals that the additional lateral information introduced by the TO framework can be effectively exploited to compute more accurate estimates of cardiac motion. Moreover among the considered algorithms the proposed APBE motion estimator regularly produced the estimates with the smallest mean error and the smallest variance, which reveals a superior accuracy and reliability. As shown by Fig. 4(a) and (b), the higher accuracy of the APBE method in the lateral direction was observed at almost every time instant.

From what concerns errors in the axial direction, as shown by Table II and Fig. 4(c) and (d), on one side both TPBE and PhCorr algorithms performed very similarly to BM. This is easily explained by the fact that lateral oscillations do not modify substantially the axial profile of the system PSF and hence no improvement is expected for the motion computation in that direction. On the other side the proposed APBE estimator was observed to produce in average slightly higher errors than the other algorithm considered. Note otherwise that, despite an increase in the average error, the error dispersion obtained by the proposed algorithm was still the smallest among the considered techniques. As a remark note that the fact that errors are much higher in the axial direction than in the lateral one is explained by the fact that, as mentioned in Section IV-B, axial displacement on RF data can be easily one order of magnitude larger than lateral ones (few pixels versus tenth of pixels). 


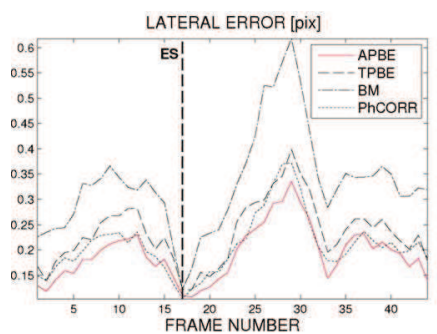

(a)

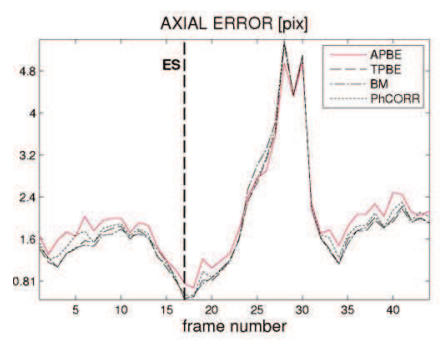

(c)

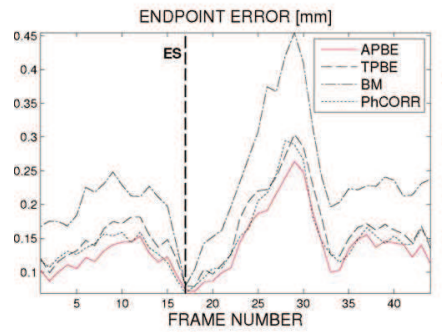

(e)

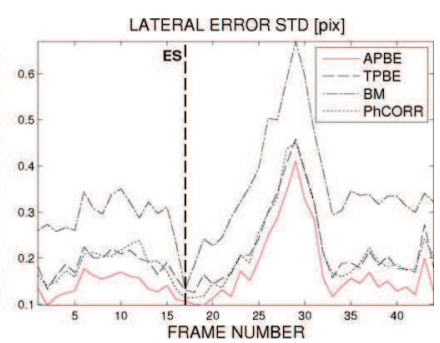

(b)

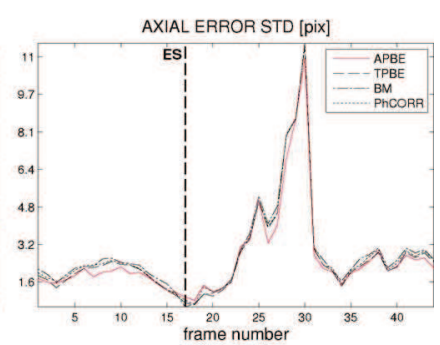

(d)

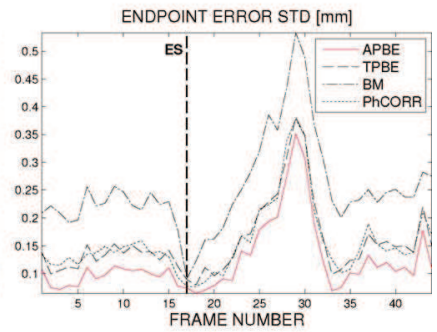

(f)
Fig. 4. Errors in the estimated displacement displayed over time for the SAx3 sequence. First, second, and third row report the lateral error (in pixel), the axial error (in pixel) and the endpoint error after scan conversion (in millimeters), respectively. Mean error and its standard deviation are presented in adjacent subfigures. Each simulated cardiac cycle goes from end diastole to end diastole. On each error plot end systolic frame is illustrated by a vertical dashed line. For a more detailed understanding of the timing cf. the M-mode of the sequence in Fig. 1(c).

The error after scan conversion is illustrated in Table III and Fig. 4(e) and (f). Clearly, is the value of displacement computed after scan conversion to represent the actual deformation of the cardiac muscle in the physical space and hence to have a meaning in the diagnostic process. In this case the proposed algorithm is the one returning the displacement estimates with the smallest mean error and standard deviation. Again this property is persistently observed at almost all time instants.

Concerning statistical consistency, all differences reported in this section were find to be significant with $p<0.0001$ as defined by the Friedman rank test. Note that spatial subsampling of the displacement field was performed prior to the statistical analysis in order to avoid correlation between samples.

Clearly, the value of the measured errors is correlated with the velocity profile during the cardiac cycle: large errors are expected in the instants of fastest motion as ejection and rapid inflow while smaller errors are expected when the motion is slow as at end systole and end diastole. This explains the bi-modal behavior of the error curves in Fig. 4. To give better insights on

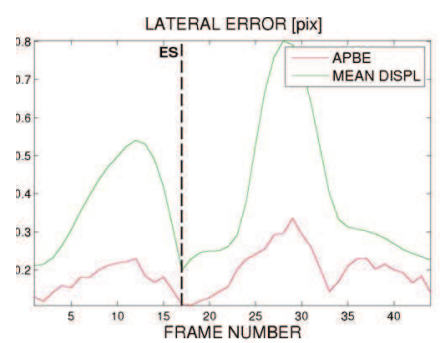

(a)

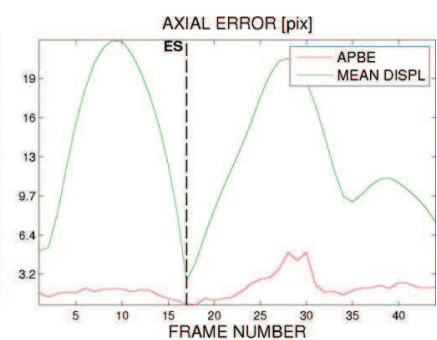

(b)

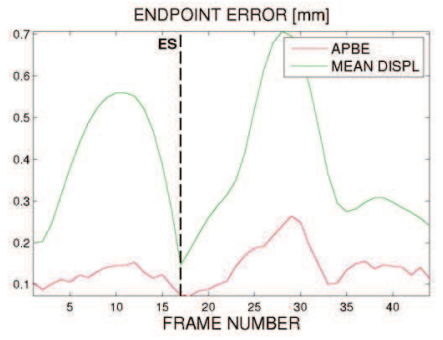

(c)

Fig. 5. Comparison between the errors computed by the proposed algorithm (in red) and the mean value of the reference displacement field (in green). Figures (a), (b), and (c) correspond respectively to the errors in the lateral direction, axial direction and after scan conversion as reported in Fig. 4(a), (c), and (e).

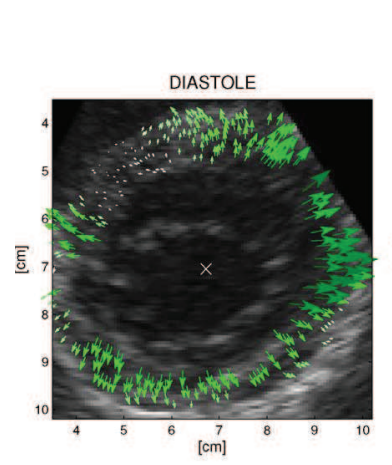

(a)

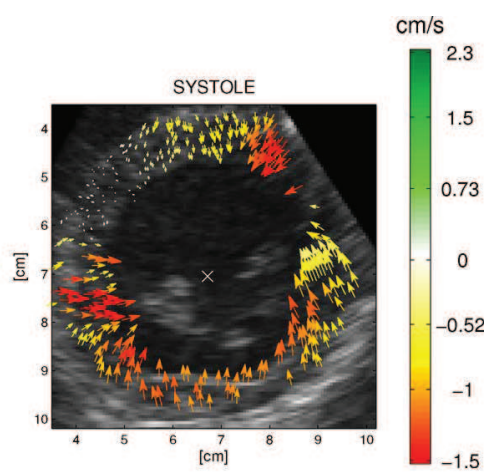

(b)
Fig. 6. Example of estimated motion fields on one diastolic (a) and one systolic (b) frame of the short axis sequence. Color encodes the radial velocity component according to the colormap in (c). White cross denotes the LV center here located manually. Note how the estimated motion fields reflects the physiological expansion and contraction of the cardiac muscle in these two phases of the cardiac cycle.

this dependency, the error curves obtained with the proposed algorithm are put in relation with the average true displacement of the cardiac muscle in Fig. 5.

The spatial behavior of the estimation error for the four approaches considered is reported in Fig. 7. The error image is relative to the 27 th frame of the simulated SAx3 sequence and illustrates the performance of each algorithm in a worst case scenario. Indeed at that instant, belonging to the rapid ventricular filling phase [cf. Fig. 1(c)], the highest average velocity over the entire cardiac cycle was measured. The error maps confirm that all estimators based on TO RF images outperform block matching in estimating lateral displacements while the precision in the axial direction is very similar among the four solutions. Among the four estimators, the APBE algorithm produces the errors with the smallest average value and the more 


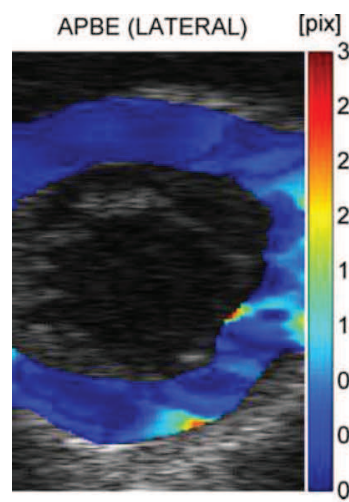

(a)

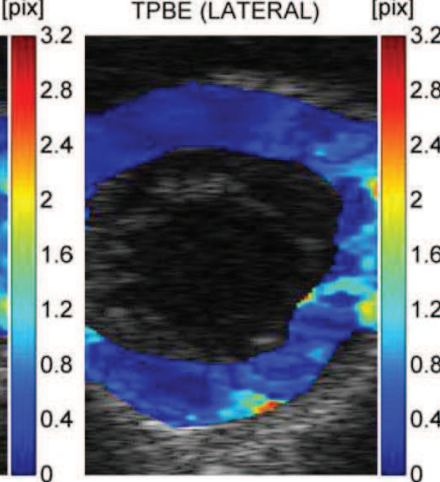

(b)

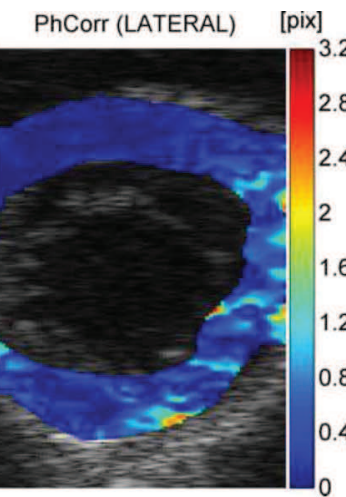

(c)

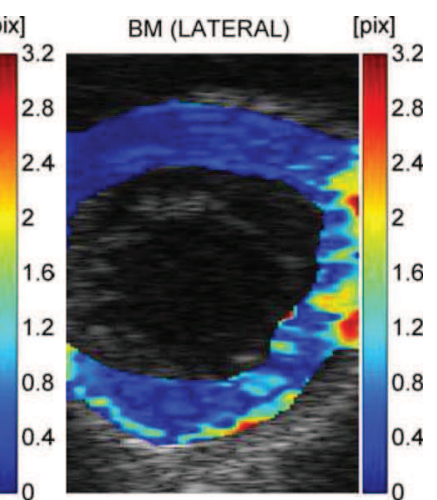

(d)

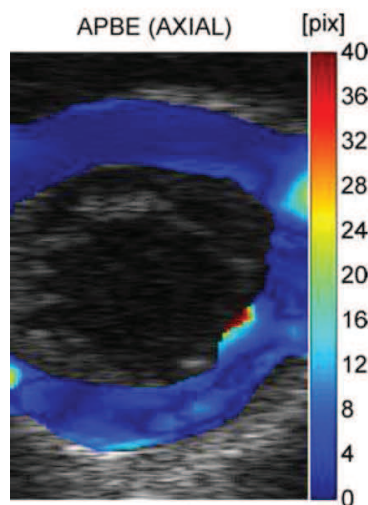

(e)

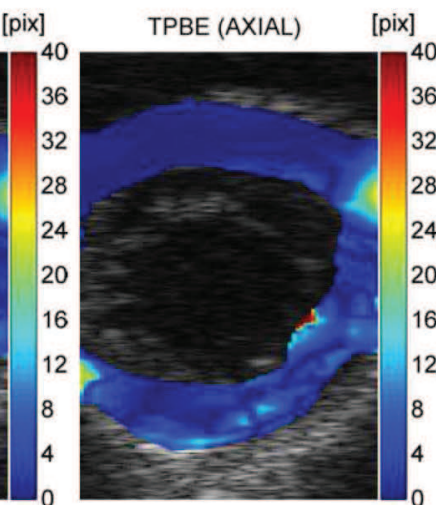

(f)

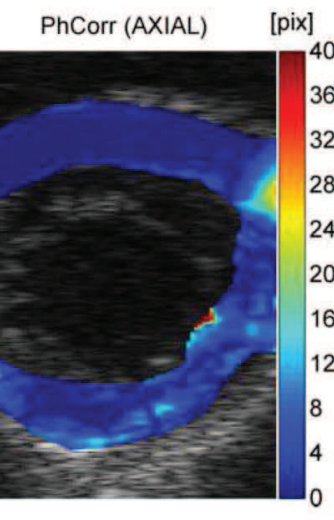

(g)

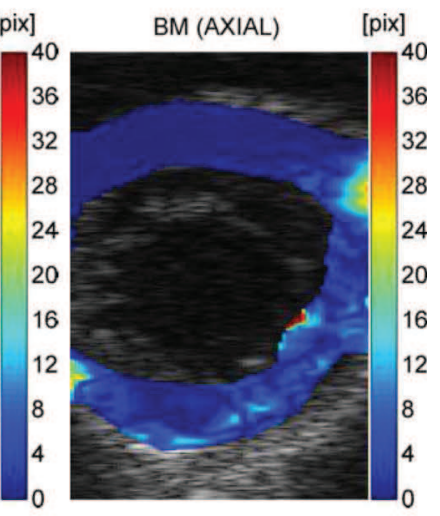

(h)

Fig. 7. Error images on frame 27 (maximum error) of the SAx3 sequence for the four considered algorithms: lateral error and axial error in the top and bottom row respectively. All errors reported in pixels. The sub-captions report the mean error \pm its standard deviation. (a) err $=0.33 \pm 0.41$. (b) err $=0.40 \pm 0.46$. (c) $\operatorname{err}=0.38 \pm 0.45$. (d) err $=0.62 \pm 0.67$. (e) err $=4.45 \pm 8.55$. (f) err $=4.37 \pm 8.61$. (g) err $=4.39 \pm 8.63$. (h) err $=4.38 \pm 8.63$.

TABLE IV

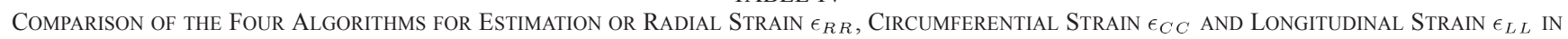
Terms of Correlation Coefficient, Bland-Altman Bias $\mu$ And Bland-Altman Limits of Agreement $\sigma$. The $p$-Value Between Brackets Reports the Statistical Significance of the Reported Value. Symbol * Denotes Values Statistically Different $(p<0.05)$ From the One of APBE. Statistical Significance of the Differences Was Measured With the Fisher's Z-VALUe For $\rho$ AND With A T-TeSt FOR $\mu$

\begin{tabular}{|c|c|c|c|c|c|c|c|c|c|}
\hline & \multicolumn{3}{|c|}{$\epsilon_{R R}$} & \multicolumn{3}{|c|}{$\epsilon_{C C}$} & \multicolumn{3}{|c|}{$\epsilon_{L L}$} \\
\hline & $\rho(p$-value $)$ & $\mu(p$-value $)$ & $\sigma$ & $\rho(p$-value $)$ & $\mu(p$-value $)$ & $\sigma$ & $\rho$ ( $p$-value $)$ & $\mu(p$-value $)$ & $\sigma$ \\
\hline APBE & $0.96(<0.001)$ & $0.24(0.71)$ & 6.60 & $0.82(0.000)$ & $1.43(0.72)$ & 5.00 & $0.94(0.000)$ & $-0.03(<0.001)$ & 1.29 \\
\hline TPBE & $0.94(<0.001)$ & $1.04(0.13)$ & 6.71 & $0.85(0.000)$ & $2.06(<0.001)$ & 4.10 & $0.93(0.000)$ & $0.02^{\star}(<0.001)$ & 1.44 \\
\hline $\mathrm{BM}$ & $0.64^{\star}(<0.001)$ & $12.41^{\star}(<0.001)$ & 25.37 & $0.50^{\star}(0.008)$ & $5.47^{\star}(<0.001)$ & 9.47 & $0.088^{\star}(0.972)$ & $3.29^{\star}(0.002)$ & 6.63 \\
\hline PhCorr & $0.87^{\star}(<0.001)$ & $-0.69(0.66)$ & 10.45 & $0.81(0.002)$ & $2.42^{\star}(<0.001)$ & 5.11 & $0.86^{\star}(0.002)$ & $0.86^{\star}(0.003)$ & 1.83 \\
\hline
\end{tabular}

uniform spatial distribution. Note that the maximum error localized on the endocardial contour is due to the motion of the mitral valve which interferes on the displacement computation within the muscle.

For what concerns the BM algorithm, sub-pixel accuracy was obtained in the lateral direction by interpolating of a factor 6 while no interpolation was employed in the axial direction. We verified that no relevant improvement in the motion estimation accuracy was obtained by increasing those values.

2) Strain Analysis: Table IV compares the four algorithms in terms of their accuracy in the computation of cardiac strain. Multiple frames are considered in order to have a statistically relevant comparison. All algorithms exploiting TO were observed to return more accurate strain estimates for all the three directions. The proposed APBE algorithm was the one producing in average more consistent estimates: it produced the highest correlation for the two strain components $\epsilon_{R R}$ and $\epsilon_{L L}$, the smallest bias for the two components $\epsilon_{R R}$ and $\epsilon_{C C}$ and the smallest standard deviation for $\epsilon_{L L}$. In the remaining cases the TPBE algorithm was the most accurate, however note that in those cases the differences with APBE were not statistically significant, except for the bias of $\epsilon_{L L}$. Moreover note that measured biases for the APBE algorithm were not statistically significant for $\epsilon_{R R}(p=0.71)$ and $\epsilon_{C C}(p=0.72)$. The Bland-Altman plot of the four algorithms considered for the radial, circumferential and longitudinal strain components respectively are illustrated in Figs. 8, 9, and 10, respectively.

Examples of computed strain curves are provided in Fig. 11. All the three algorithms exploiting TO produce strain curves closer to the benchmark than BM. We measured the normalized 


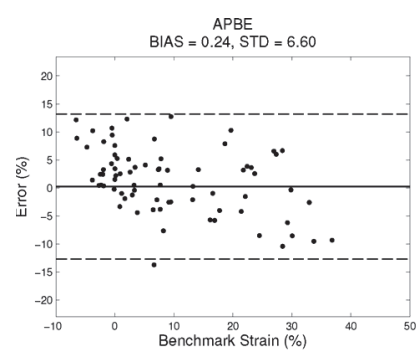

(a)

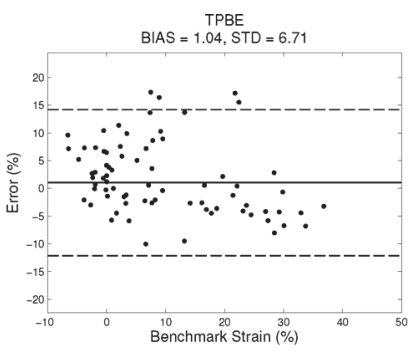

(b)

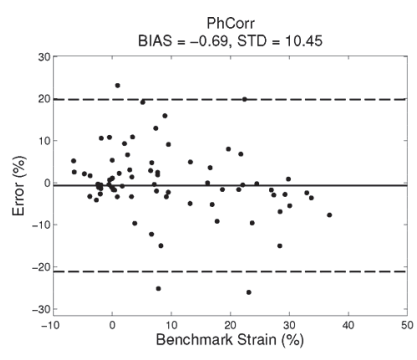

(c)

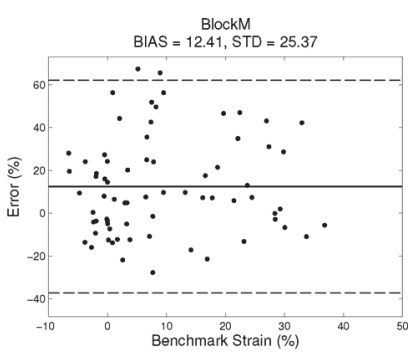

(d)

Fig. 8. Bland-Altman plot for the radial strain component $\epsilon_{R R}$. Horizontal line in each plot represents the bias $\mu$ while the two dashed lines represent the limits of agreement $[\mu-1.96 \sigma ; \mu+1.96 \sigma]$.

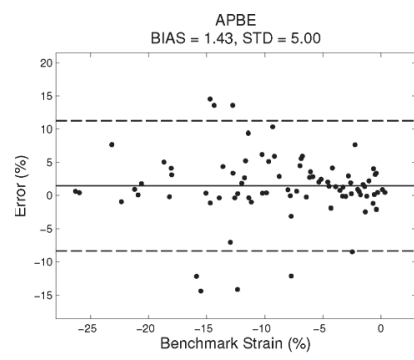

(a)

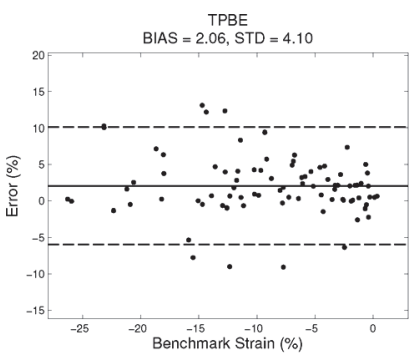

(b)

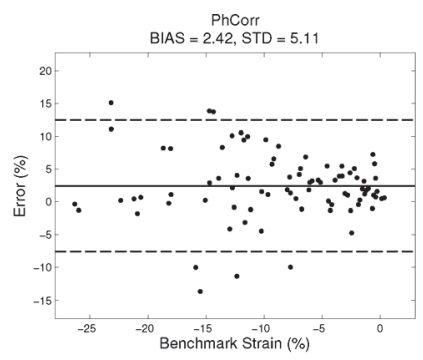

(c)

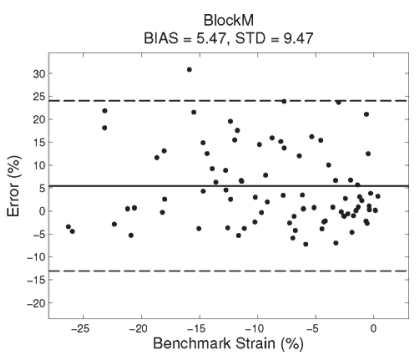

(d)

Fig. 9. Bland-Altman plot for the circumferential strain component $\epsilon_{C C}$. Horizontal line in each plot represents the bias $\mu$ while the two dashed lines represent the limits of agreement $[\mu-1.96 \sigma ; \mu+1.96 \sigma]$.

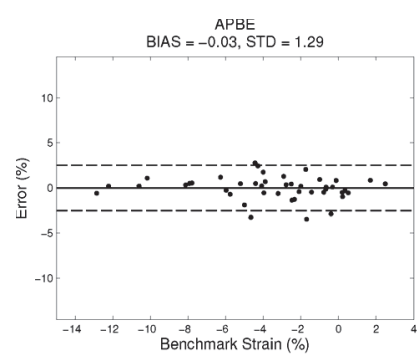

(a)

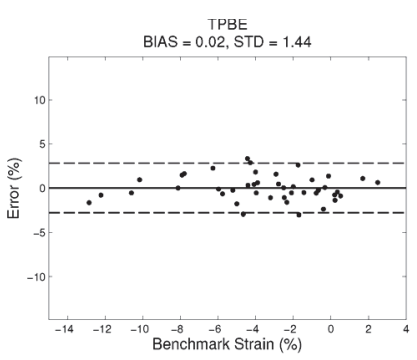

(b)

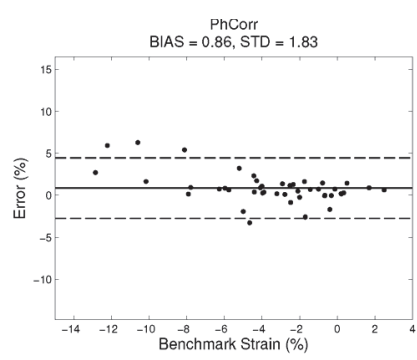

(c)

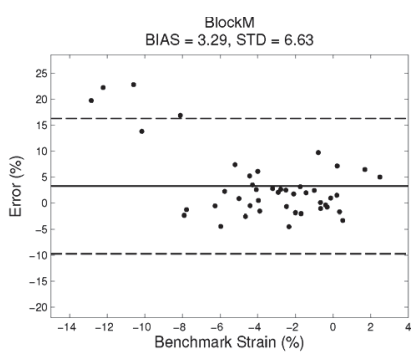

(d)

Fig. 10. Bland-Altman plot for the longitudinal strain component $\epsilon_{L L}$. Horizontal line in each plot represents the bias $\mu$ while the two dashed lines represent the limits of agreement $[\mu-1.96 \sigma ; \mu+1.96 \sigma]$.

distance between the estimated strain curve and the benchmark as

$$
D_{\text {algo }}=\sqrt{\sum_{k} \frac{(\epsilon(k)-\bar{\epsilon}(k))^{2}}{\bar{\epsilon}(k)^{2}}}
$$

where $\bar{\epsilon}(k)$ is the benchmark global strain at time $k$ while $\epsilon(k)$ is the computed one. For all strain components, the APBE algorithm returned the estimate with the smallest normalized distance: for $\epsilon_{R R}$ it was $D_{\mathrm{APBE}}=0.19, D_{\mathrm{TPBE}}=0.23$, $D_{\mathrm{PhCorr}}=0.36$ and $D_{\mathrm{BM}}=0.85$; for $\epsilon_{C C}$ it was $D_{\mathrm{APBE}}=0.10, D_{\mathrm{TPBE}}=0.16, D_{\mathrm{PhCorr}}=0.27$ and $D_{\mathrm{BM}}=0.67$; for $\epsilon_{L L}$ it was $D_{\mathrm{APBE}}=0.05, D_{\mathrm{TPBE}}=0.07$, $D_{\mathrm{PhCorr}}=0.91$ and $D_{\mathrm{BM}}=0.25$.

End-systolic strain values have been shown to be relevant for the assessment of cardiac function. Given the size of the data set, the number of end-systolic strain values were not sufficient to have statistical significance. The results of the strain analysis restricted to end-systole are reported for sake of completeness in Table V. What qualitatively shown is that also in this case techniques based on TO in average outperform BM (cf. biases and standard deviations). Comparing the different phase-based techniques becomes instead more complicated given the very limited number of samples.

3) Computational Complexity: A final issue concerns the computational complexity. Fast processing is indeed particularly desirable as far as medical ultrasound is concerned, since the real-time capability is one of the main advantages of this technique. All the considered algorithms were implemented in MATLAB (R2011b, The Math-Works, Natick, MA, USA) and executed on a desktop PC with a $3.47 \mathrm{GHz}$ Intel Xeon X5690 processor, $12 \mathrm{~Gb}$ of RAM and running Windows 7 . The RF image size was of $4562 \times 112$ pixels $^{2}$ for the SAx sequence and $6724 \times 189$ pixels $^{2}$ for the A4C.

The most onerous step was the block-matching initialization, which on the SAx sequence took roughly $60 \mathrm{~s} /$ frame. 
TABLE V

COMPARison OF the Four Algorithms for Strain Estimation. Correlation Coefficient, Bland-Altman Bias $\mu$ And Bland-Altman Limits of Agreement $\sigma$. Strain Values at End Systole Are Considered Only

\begin{tabular}{|c|c|c|c|c|c|c|c|c|c|}
\hline & \multicolumn{3}{|c|}{$\epsilon_{R R}$} & \multicolumn{3}{|c|}{$\epsilon_{C C}$} & \multicolumn{3}{|c|}{$\epsilon_{L L}$} \\
\hline & $\rho$ ( $p$-value $)$ & $\mathrm{BA} \mu$ & BA $\sigma$ & $\rho$ & BA $\mu$ & BA $\sigma$ & $\rho$ ( $p$-value $)$ & $\mathrm{BA} \mu$ & BA $\sigma$ \\
\hline APBE & $0.94(0.001)$ & -0.22 & 9.79 & $0.95(0.00)$ & 1.58 & 7.37 & $0.86(0.14)$ & -0.13 & 2.55 \\
\hline TPBE & $0.91(0.001)$ & -0.30 & 8.64 & $0.97(0.00)$ & 2.57 & 5.53 & $0.87(0.13)$ & 0.12 & 2.89 \\
\hline $\mathrm{BM}$ & $0.92(0.003)$ & 4.50 & 25.11 & $0.85(0.00)$ & 4.94 & 9.88 & $0.76(0.24)$ & 5.17 & 9.99 \\
\hline PhCorr & $0.90(0.003)$ & -4.66 & 14.81 & $0.91(0.00)$ & 3.01 & 7.45 & $0.90(0.09)$ & 0.64 & 2.29 \\
\hline
\end{tabular}

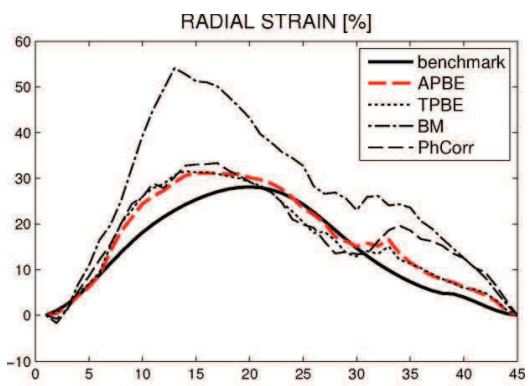

(a)

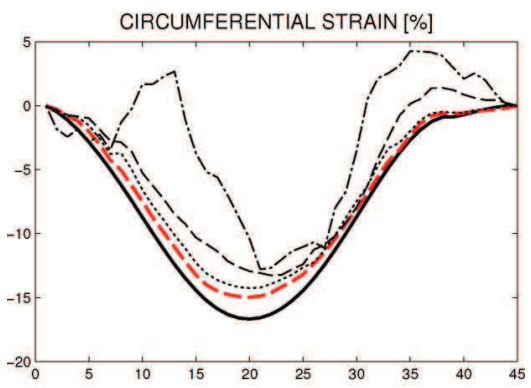

(b)

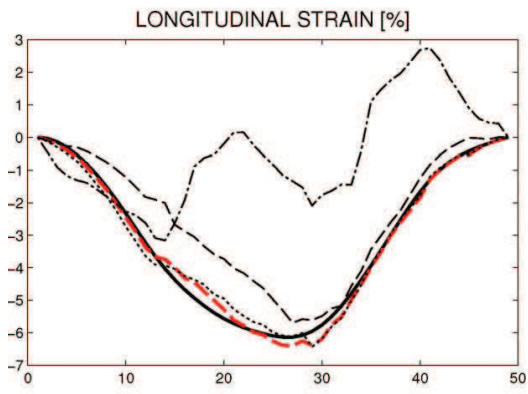

(c)

Fig. 11. Comparison among the four computed strain curves and the benchmark. Global strain values (i.e., averaged over the entire muscle) are considered at each time instant.

This is clearly a limitation of the current implementation. Nevertheless one should consider that real-time implementations of speckle-tracking exist and can be directly employed to speed up the initialization procedure [67]. Concerning the refinement instead, this took roughly $2.5 \mathrm{~s} /$ frame for the phasebased estimators and $22 \mathrm{~s} /$ frame for BM. Again, the reported times are certainly implementation dependent. In particular more effective implementation can be adopted to decrease the cost associated to BM. Nonetheless it is important to note the computational complexity of the phase based estimators is considerably inferior to the one associated to BM. Indeed, in the

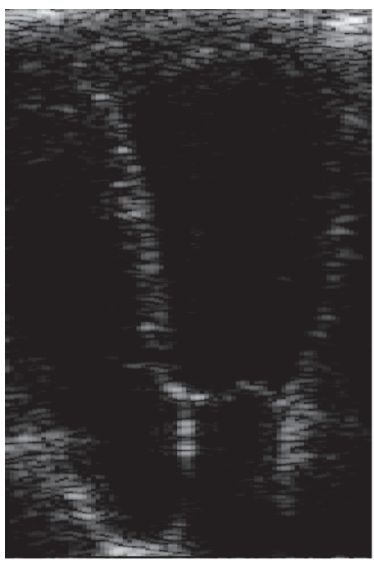

(a)

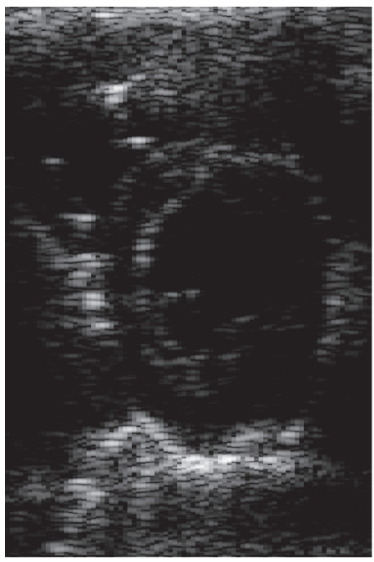

(c)

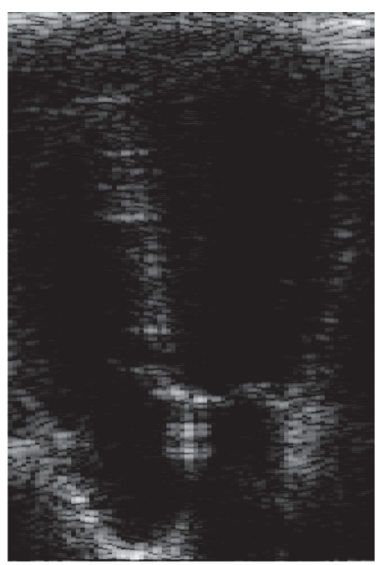

(b)

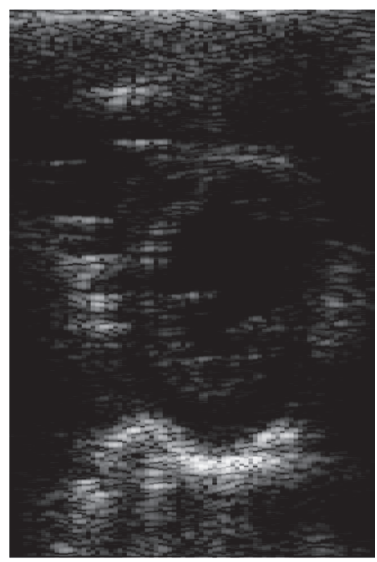

(d)
Fig. 12. Sample images acquired with the UlaOp platform. Two acquisition modalities on an apical view are compared in (a) and (b). The two acquisition modalities on an short axis view are compared in (c) and (d). (a) Without TO. (b) With TO. (c) Without TO. (d) With TO.

first case, the displacement is directly given by the solution of the two $3 \times 3$ linear systems of equations given by the least squares solution of (10) and (11), while BM requires interpolation to obtain sub-pixel accuracy and the iterative search of the best match position within each block. For this reason a considerable speedup over BM can still be expected even in more optimized implementations.

\section{B. In Vivo Results}

The goal to this section is to show that the state of advance of the proposed framework is beyond simple simulation and an in vivo evaluation on real clinical recording is already possible. 


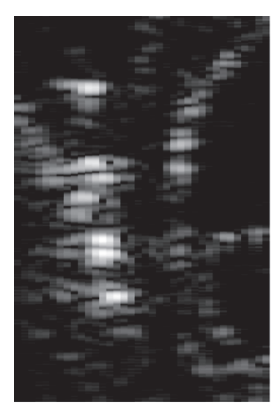

(a)

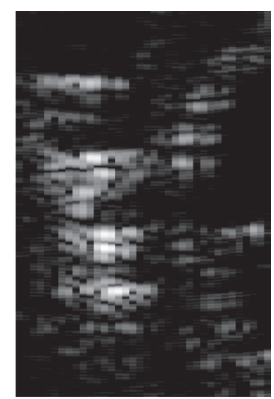

(b)
Fig. 13. Zoom of the heart septum on the short axis view without (a) and with (b) transverse oscillations.

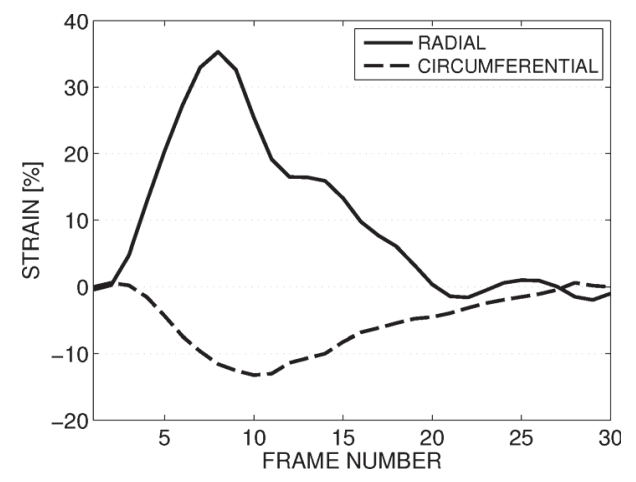

Fig. 14. Strain curves computed with the proposed APBE algorithm on the short-axis acquisition.

The proposed image formation technique with transverse oscillations was implemented on a real scanner. In particular the ultrasound research platform Ula-op [52] equipped with a cardiac probe model PA230 from Esaote (Esaote Spa, Genoa, Italy) was used. The acquisitions was performed by an experienced radiologist on one 25-year-old male healthy volunteer. In particular two views were acquired: one apical four chamber and one parasternal short axis. In order to compare standard B-mode images and the proposed TO beamforming, the RF lines of the two imaging modalities were interleaved during the acquisition: every second line corresponded to a conventional B-mode sector scan and the other one used the beamforming strategy of Section II to provide TO images. The frame rate for both modalities was of 25 frames/s. The RF signals were acquired at a sampling frequency of $50 \mathrm{MHz}$. The beam density was of 1 beam/degree. Due to memory limitations, a total of 49 frames $(2 \mathrm{~s})$ for each mode could be acquired. This was sufficient to obtain one complete cardiac cycle.

Fig. 12 shows one sample frame from each of the two views when acquired with and without TO. Fig. 13 shows a selection corresponding to the heart septum on the short axis view. From the latter the difference in speckle pattern is evident.

Both sequences were processed with the APBE algorithm of Section III. The strain curves for the radial and circumferential strain components computed from the SAx sequence are reported in Fig. 14. The measured strain values are consistent with what reported in literature for an healthy heart [68]. A thorough clinical evaluation including strain and strain rate values on healthy and pathological subjects falls beyond the scope of this paper and will be made object of future studies.

\section{Discussions AND CONCLUSION}

The paper introduced a novel setup for improving cardiac motion estimation with ultrasound. Despite the important progresses made in the field even best performing techniques still register a low accuracy in estimating displacement/strain values in the lateral direction (i.e., perpendicular to the beam propagation). The proposed framework aimed at overcoming this limitation by combining two elements: an unconventional beamforming technique and a dedicated motion estimation algorithm.

The beamformer was designed so to add oscillations in the lateral direction. As already known from blood flow imaging and elastography such an acquisition scheme leads to an image model intrinsically better suited for the estimation of lateral displacements. We then presented an algorithm specifically designed to exploit the availability of a phase information in the two directions. This was done by decomposing the ultrasound image into two 2-D single-orthant analytic signals and assuming time conservation of the two associated image phases.

A quantitative evaluation of the proposed setup was performed in silico on five synthetic cardiac ultrasound sequences. The comparison included block-matching on standard images without transverse oscillations and other two phase-based solutions exploiting the presence of oscillations in the lateral direction. The obtained results revealed an higher accuracy in the estimation of the cardiac motion when TO were employed. In particular the proposed estimator were the most accurate among the three phase-based algorithms. This better accuracy reflected into a more robust estimation of the cardiac strain. More specifically the proposed setup was the one allowing in average for the highest correlation with the reference strain values, the smallest bias and the smallest limits of agreement as computed with the Bland Altman analysis.

While the estimation of lateral displacements were improved by the employment of the affine model, leading to an overall higher accuracy on the motion field after scan-conversion, we acknowledge that the estimates in the axial direction were in average slightly less precise than what obtained with the other techniques considered. A possible reason is that a more complex model (as the affine one) is more prone to over-fitting than a simple one (as the translation one) in the presence of noise. One solution would be locally choosing for the model best adapted to the data (translation or affine in our case) as proposed e.g., in [69]. This possibility will be considered in future studies.

The synthetic evaluation is a first necessary step towards a more thorough validation including phantom experiments and real patients, which will be the topic of future studies. In this perspective an implementation of the proposed beamforming technique with transverse oscillations on the UlaOP ultrasound research platform was presented in the paper along with the preliminary motion estimation results on one healthy volunteer. In particular in this preliminary evaluation it was shown that the extracted strain curves were consistent with what expected from the literature.

An issue that requires consideration in view of a clinical evaluation is how transverse oscillations are perceived by the final user, i.e., the physician. This evaluation must consider the 
opinion of multiple experts and falls beyond the scope of this paper. Nevertheless one should consider that several possibilities exist to exploit transverse oscillations for motion/strain estimation while visualizing images close to the standard b-mode images currently of use in the clinical practice. One possibility would be to acquire the two modalities in parallel (possibly with a dedicated architecture for TO). The second possibility would be extending envelope detection to the lateral direction so to account for lateral oscillations. Interestingly this 2-D envelope could be directly obtained as the amplitude of the single-orthant analytic signal computed in (5), and hence would not require supplemental calculations.

Concerning the computational complexity, the generation of transverse oscillations only implies modifying the receive apodization function of the system and hence it does not increase the computational demand. Instead for what concerns the motion estimation algorithm its implementation in terms of computational efficiency is still sub-optimal (MATLAB implementation) and hence not competitive with the block matching implementations present on commercial systems. Nevertheless the proposed estimator is in principle less onerous than block matching as no iterative research within a search window is needed and no interpolation is needed to reach sub-pixel accuracy. Moreover, being local, the proposed estimator is intrinsically parallelizable and can hence take advantage of parallel computation platforms as GPUs.

Future studies include an extension of the proposed setup to 3-D echocardiography. Despite 2-D still remains the modality of choice in the clinical practice, 3-D US has shown to be potentially more accurate in the quantification of cardiac mechanics and, therefore, a more reliable diagnostic tool. For what concerns the proposed framework, the extension of the estimator to 3-D is straightforward, cf. [49]. For the beamforming of 3-D TO images, several approaches are possible. First of all a matrix array will be necessary because a two dimensional apodization function must be designed. Pihl and Jensen and Pihl et al. proposed in [70] and in [71] respectively a twofold 2-D approach where two different 3-D volumes are formed to estimate $3-\mathrm{D}$ vector motion maps: one with TO oriented in the lateral direction and one with the TO oriented in the elevation direction. In [72], Salles et al. proposed to use instead a separable 2-D apodization function featuring four Gaussian peaks to obtain directly volumes featuring both, lateral and elevation oscillations.

\section{ApPENDIX A}

\section{Decomposition of the Motion Estimation Problem}

For one pixel of coordinates $(x, z)$, using the analytical expression of the Jacobian matrix given in (8), the spatial phase time consistency in (7) may be further developed as a system of two equations

$$
\begin{aligned}
& \Phi_{s o 1}^{(t)}(x, z)=2 \pi / \lambda_{x} d_{1}(x, z)+2 \pi / \lambda_{z} d_{2}(x, z) \\
& \Phi_{s o 2}^{(t)}(x, z)=-2 \pi / \lambda_{x} d_{1}(x, z)+2 \pi / \lambda_{z} d_{2}(x, z) .
\end{aligned}
$$

Replacing $d_{1}(x, z)$ and $d_{2}(x, z)$ in (17) by the affine model in (9) and adding and substracting the two previous equations leads to

$$
\begin{aligned}
& \lambda_{x} / 2 \pi\left(\Phi_{\text {so } 1}^{(t)}(x, z)-\Phi_{\text {so } 2}^{(t)}(x, z)\right)=\left(\begin{array}{lll}
1 & x & z
\end{array}\right)\left(\begin{array}{l}
d_{10} \\
d_{1 x} \\
d_{1 z}
\end{array}\right) \\
& \lambda_{z} / 2 \pi\left(\Phi_{s o 1}^{(t)}(x, z)+\Phi_{s o 2}^{(t)}(x, z)\right)=\left(\begin{array}{lll}
1 & x & z
\end{array}\right)\left(\begin{array}{l}
d_{20} \\
d_{2 x} \\
d_{2 z}
\end{array}\right) .
\end{aligned}
$$

Finally, by applying (18) for a block of $N$ pixels of coordinates $\left(x_{i}, z_{i}\right)$ with $i$ running from 0 to $N-1$, we obtain the two systems of equations given in (10) and (11).

\section{ACKNOWLEDGMENT}

The authors would also like to thank F. Douziech for his participation to this work.

\section{REFERENCES}

[1] World Health Org., World Health Stat. 2012.

[2] T. P. Abraham, V. L. Dimaano, and H.-Y. Liang, "Role of tissue Doppler and strain echocardiography in current clinical practice," Circulation, vol. 116, no. 22, pp. 2597-2609, 2007.

[3] C. Cottrell and J. N. Kirkpatrick, "Echocardiographic strain imaging and its use in the clinical setting," Exp. Rev. Cardiovasc. Ther., vol. 8, no. 1, pp. 93-102, 2010.

[4] J. D'Hooge, E. Konofagou, F. Jamal, A. Heimdal, L. Barrios, B. Bijnens, J. Thoen, F. Van de Werf, G. Sutherland, and P. Suetens, "Two-dimensional ultrasonic strain rate measurement of the human heart in vivo," IEEE Trans. Ultrason. Ferroelectr. Freq. Control, vol. 49, no. 2, pp. 281-286, Feb. 2002.

[5] A. M. Shah and S. D. Solomon, "Myocardial deformation imaging: Current status and future directions," Circulation, vol. 125, no. 2, pp. e244-e248, 2012.

[6] G. R. Sutherland, G. Di Salvo, P. Claus, J. D’Hooge, and B. Bijnens, "Strain and strain rate imaging: A new clinical approach to quantifying regional myocardial function," J. Am. Soc. Echocardiogr., vol. 17, no. 7, pp. 788-802, 2004.

[7] G. E. Mailloux, A. Bleau, M. Bertrand, and R. Petitclerc, "Computer analysis of heart motion from two-dimensional echocardiograms," IEEE Trans. Biomed. Eng., vol. 34, no. 5, pp. 356-364, May 1987.

[8] M. De Craene, G. Piella, O. Camara, N. Duchateau, E. Silva, A. Doltra, J. Dhooge, J. Brugada, M. Sitges, and A. F. Frangi, "Temporal diffeomorphic free-form deformation: Application to motion and strain estimation from 3D echocardiography," Med. Image Anal., vol. 16, no. 2, pp. 427-450, 2012.

[9] B. Heyde, R. Jasaityte, D. Barbosa, V. Robesyn, S. Bouchez, P. Wouters, F. Maes, P. Claus, and J. D'Hooge, "Elastic image registration versus speckle tracking for 2-d myocardial motion estimation: A direct comparison in vivo," IEEE Trans. Med. Imag., vol. 32, no. 2, pp. 449-459, Feb. 2013.

[10] M. Alessandrini, H. Liebgott, D. Barbosa, and O. Bernard, "Monogenic phase based optical flow computation for myocardial motion analysis in 3-D echocardiography," in Statistical Atlases and Computational Models of the Heart. Imaging and Modelling Challenges. Berlin, Germany: Springer, 2013, vol. 7746, Lecture Notes in Computer Science, ch. 19, pp. 159-168.

[11] K. McLeod, A. Prakosa, T. Mansi, M. Sermesant, and X. Pennec, "An incompressible log-domain demons algorithm for tracking heart tissue," in Statistical Atlases and Computational Models of the Heart. Imaging and Modelling Challenges. Berlin, Germany: Springer, 2012, vol. 7085, Lecture Notes in Computer Science, ch. 6, pp. 55-67. 
[12] M. De Craene, S. Marchesseau, B. Heyde, H. Gao, M. Alessandrini, O. Bernard, G. Piella, A. Porras, L. Tautz, A. Hennemuth, A. Prakosa, H. Liebgott, O. Somphone, P. Allain, S. M. Ebeid, H. Delingette, M. Sermesant, J. D'Hooge, and E. Saloux, "3d strain assessment in ultrasound (STRAUS): A synthetic comparison of five tracking methodologies," IEEE Trans. Med. Imag., vol. 32, no. 9, pp. 1632-1646, Sep. 2013.

[13] B. Touil, A. Basarab, P. Delachartre, O. Bernard, and D. Friboulet, "Analysis of motion tracking in echocardiographic image sequences: Influence of system geometry and point-spread function," Ultrasonics, vol. 50 , no. 3, pp. 373-386, 2010

[14] P. Baraldi, A. Sarti, C. Lamberti, A. Prandini, and F. Sgallari, "Evaluation of differential optical flow techniques on synthesized echo images," IEEE Trans. Biomed. Eng., vol. 43, no. 3, pp. 259-272, Mar. 1996.

[15] G. E. Mailloux, F. Langlois, P. Simard, and M. Bertrand, "Restoration of the velocity field of the heart from two-dimensional echocardiograms," IEEE Trans. Med. Imag., vol. 8, no. 2, pp. 143-153, Jun. 1989.

[16] V. Behar, D. Adam, P. Lysyansky, and Z. Friedman, "The combined effect of nonlinear filtration and window size on the accuracy of tissue displacement estimation using detected echo signals," Ultrasonics, vol. 41, no. 9, pp. 743-753, 2004.

[17] M. Suhling, M. Arigovindan, C. Jansen, P. Hunziker, and M. Unser, "Myocardial motion analysis from b-mode echocardiograms," IEEE Trans. Image Process., vol. 14, no. 4, pp. 525-536, Apr. 2005.

[18] M. Alessandrini, A. Basarab, H. Liebgott, and O. Bernard, "Myocardial motion estimation from medical images using the monogenic signal," IEEE Trans. Image Process., vol. 22, no. 3, pp. 1084-1095, Mar. 2013.

[19] V. Behar, D. Adam, P. Lysyansky, and Z. Friedman, "Improving motion estimation by accounting for local image distortion," Ultrasonics, vol. 43, no. 1, pp. 57-65, 2004.

[20] Q. Duan, E. Angelini, S. Homma, and A. Laine, "Validation of optical-flow for quantification of myocardial deformations on simulated RT3D ultrasound," in Proc. 4th IEEE Int. Symp. Biomed. Imag.: From Nano to Macro2007, 2007, pp. 944-947.

[21] C. Kontogeorgakis, M. Strintzis, N. Maglaveras, and I. Kokkinidis, "Tumor detection in ultrasound b-mode images through motion estimation using a texture detection algorithm," in Comput. Cardiol., 1994, pp. 117-120.

[22] F. Yeung, S. F. Levinson, and K. J. Parker, "Multilevel and motion model-based ultrasonic speckle tracking algorithms," Ultrasound Med. Biol., vol. 24, no. 3, pp. 427-442, 1998.

[23] M. Strintzis and I. Kokkinidis, "Maximum likelihood motion estimation in ultrasound image sequences," IEEE Signal Process. Lett., vol. 4, no. 6, pp. 156-157, Jun. 1997

[24] Y. Yue, J. Clark, and D. Khoury, "Speckle tracking in intracardiac echocardiography for the assessment of myocardial deformation," IEEE Trans. Biomed. Eng., vol. 56, no. 2, pp. 416-425, Feb. 2009.

[25] B. Cohen and I. Dinstein, "New maximum likelihood motion estimation schemes for noisy ultrasound images," Pattern Recognit., vol. 35, no. 2, pp. 455-463, 2002.

[26] M. Linguraru, N. Vasilyev, P. del Nido, and R. Howe, "Fast block flow tracking of atrial septal defects in $4 \mathrm{~d}$ echocardiography," Med. Image Anal., vol. 12, no. 4, pp. 397-412, 2008.

[27] M. Ledesma-Carbayo, J. Kybic, M. Desco, A. Santos, M. Suhling, P. Hunziker, and M. Unser, "Spatio-temporal nonrigid registration for ultrasound cardiac motion estimation," IEEE Trans. Med. Imag., vol. 24, no. 9, pp. 1113-1126, Sep. 2005.

[28] J. Woo, B.-W. Hong, C.-H. Hu, K. K. Shung, C. C. J. Kuo, and P. Slomka, "Non-rigid ultrasound image registration based on intensity and local phase information," J. Signal Process. Syst., vol. 54, no. 1-3, pp. 33-43, 2009.

[29] A. Elen, C. H. Fai, D. Loeckx, G. Hang, P. Claus, P. Suetens, F. Maes, and J. D'Hooge, "Three-dimensional cardiac strain estimation using spatio-temporal elastic registration of ultrasound images: A feasibility study," IEEE Trans. Med. Imag., vol. 27, no. 11, pp. 1580-1591, Nov. 2008.

[30] A. Pesavento, C. Perrey, M. Krueger, and H. Ermert, "A timeefficient and accurate strain estimation concept for ultrasonic elastography using iterative phase zero estimation," IEEE Trans. Ultrason., Ferroelectr. Freq. Control, vol. 46, no. 5, pp. 1057-1067, Sep. 1999.
[31] M. Lubinski, S. Emelianov, and M. ODonnell, "Speckle tracking methods for ultrasonic elasticity imaging using short-time correlation," IEEE Trans. Ultrason., Ferroelectr. Freq. Control, vol. 46, no. 1, pp. 82-96, Jan. 1999.

[32] H. Chen, H. Shi, and T. Varghese, "Improvement of elastographic displacement estimation using a two-step cross-correlation method," $U l-$ trasound Med. Biol., vol. 33, no. 1, pp. 48-56, 2007.

[33] J. D’Hooge, B. Bijnens, J. Thoen, F. Van de Werf, G. Sutherland, and P. Suetens, "Echocardiographic strain and strain-rate imaging: A new tool to study regional myocardial function," IEEE Trans. Med. Imag., vol. 21, no. 9, pp. 1022-1030, Sep. 2002.

[34] R. G. P. Lopata, M. M. Nillesen, H. H. G. Hansen, I. H. Gerrits, J. M. Thijssen, and C. L. de Korte, "Performance evaluation of methods for two-dimensional displacement and strain estimation using ultrasound radio frequency data," Ultrasound Med. Biol., vol. 35, no. 5, pp. 796-812, 2009.

[35] R. G. Lopata, M. M. Nillesen, J. M. Thijssen, L. Kapusta, and C. L. de Korte, "Three-dimensional cardiac strain imaging in healthy children using RF-data," Ultrasound Med. Biol., vol. 37, no. 9, pp. 1399-1408, 2011.

[36] E. Konofagou and J. Ophir, "A new elastographic method for estimation and imaging of lateral displacements, lateral strains, corrected axial strains and Poissons ratios in tissues," Ultrasound Med. Biol., vol. 24 , no. 8, pp. 1183-1199, 1998

[37] J. Luo and E. Konofagou, "High-frame rate, full-view myocardial elastography with automated contour tracking in murine left ventricles in vivo," IEEE Trans. Ultrason., Ferroelectr. Freq. Control, vol. 55, no. 1, pp. 240-248, Jan. 2008.

[38] W. Yu, P. Yan, A. Sinusas, K. Thiele, and J. Duncan, "Towards pointwise motion tracking in echocardiographic image sequences-Comparing the reliability of different features for speckle tracking," Med. Image Anal., vol. 10, no. 4, pp. 495-508, 2006.

[39] M. A. Lubinski, S. Y. Emelianov, K. R. Raghavan, A. E. Yagle, A. R. Skovoroda, and M. O'Donnell, "Lateral displacement estimation using tissue incompressibility," IEEE Trans. Ultrason., Ferroelectr. Freq. Control, vol. 43, no. 2, pp. 247-256, Mar. 1996.

[40] M. Aderson, "Multi-dimensional velocity estimation with ultrasound using spatial quadrature," IEEE Trans. Ultrason., Ferroelectr. Freq. Control, vol. 45, no. 3, pp. 852-861, May 1998.

[41] J. Jensen and P. Munk, "A new method for estimation of velocity vectors," IEEE Trans. Ultrason., Ferroelectr. Freq. Control, vol. 45, no. 3, pp. 837-851, May 1998.

[42] H. Liebgott, J. Wilhjehm, J. Jensen, D. Vray, and P. Delachartre, "PSF dedicated to estimation of displacement vectors for tissue elasticity imaging with ultrasound," IEEE Trans. Ultrason., Ferroelectr. Freq. Control, vol. 54, no. 4, pp. 746-756, Apr. 2007.

[43] C. Sumi, "Displacement vector measurement using instantaneous ultrasound signal phase-multidimensional autocorrelation and Doppler methods," IEEE Trans. Ultrason., Ferroelectr. Freq. Control, vol. 55, no. 1 , pp. 24-43, Jan. 2008.

[44] X. Guo, D. Friboulet, and H. Liebgott, "Transverse oscillations beamformer design for sector scan using back-propagation," in Proc. 9th IEEE Int. Symp. Biomed. Imag., May 2012, pp. 1100-1103.

[45] H. Liebgott, A. Basarab, S. Marincas, O. Bernard, and D. Friboulet, "Tangential oscillations for motion estimation in echocardiography," in Proc. IEEE Ultrason. Symp., Nov. 2008, pp. 1761-1764.

[46] H. Liebgott, A. B. Salem, A. Basarab, H. Gao, P. Claus, J. D'Hooge, P. Delachartre, and D. Friboulet, "Tangential sound field oscillations for 2D motion estimation in echocardiography," in Proc. IEEE Int. Ultrason. Symp., Sep. 2009, pp. 498-501.

[47] M. Alessandrini, H. Liebgott, D. Friboulet, and O. Bernard, "Monogenic phase based myocardial motion analysis from cardiac ultrasound with transverse oscillations," in Proc. IEEE Int. Ultrason. Symp., Oct. 2012, pp. 1098-1101.

[48] M. Alessandrini, H. Liebgott, D. Friboulet, and O. Bernard, "Simulation of realistic echocardiographic sequences for ground-truth validation of motion estimation," in Proc. 19th IEEE Int. Conf. Image Process., 2012, pp. 2329-2332.

[49] A. Basarab, H. Liebgott, and P. Delachartre, "Analytic estimation of subsample spatial shift using the phases of multidimensional analytic signals," IEEE Trans. Image Process., vol. 18, no. 2, pp. 440-447, Feb. 2009 
[50] L. Bohs and G. Trahey, "A novel method for angle independent ultrasonic imaging of blood flow and tissue motion," IEEE Trans. Biomed. Eng., vol. 38, no. 3, pp. 280-286, Mar. 1991.

[51] W.-N. Lee, Z. Qian, C. L. Tosti, T. R. Brown, D. N. Metaxas, and E. E. Konofagou, "Preliminary validation of angle-independent myocardial elastography using MR tagging in a clinical setting," Ultrasound Med. Biol., vol. 34, no. 12, pp. 1980-1997, 2008.

[52] P. Tortoli, L. Bassi, E. Boni, A. Dallai, F. Guidi, and S. Ricci, "ULAOP: An advanced open platform for ultrasound research," IEEE Trans. Ultrason., Ferroelectr. Freq. Control, vol. 56, no. 10, pp. 2207-2216, Oct. 2009.

[53] H. Liebgott, A. Basarab, P. Gueth, D. Friboulet, and P. Delachartre, "Transverse oscillations for tissue motion estimation," Ultrasonics, vol. 50, no. 6, pp. 548-555, 2010.

[54] J. W. Goodman, Introduction to Fourier Optics, 3rd ed. Greenwood Village, CO: Roberts Company Publishers, Dec. 2004.

[55] K. Hansen, J. Udesen, C. Thomsen, J. Jensen, and M. Nielsen, "In vivo validation of a blood vector velocity estimator with MR angiography," IEEE Trans. Ultrason., Ferroelectr. Freq. Control, vol. 56, no. 1, pp. 91-100, Jan. 2009.

[56] J. D’Hooge, A. Heimdal, F. Jamal, T. Kukulski, B. Bijnens, F. Rademakers, L. Hatle, P. Suetens, and G. R. Sutherland, "Regional strain and strain rate measurements by cardiac ultrasound: Principles, implementation and limitations," Eur. J. Echocardiogr., vol. 1, no. 3, pp. 154-170, Sep. 2000.

[57] T. Bulow and G. Sommer, "Hypercomplex signals-A novel extension of the analytic signal to the multidimensional case," IEEE Trans. Signal Process., vol. 49, no. 11, pp. 2844-2852, Nov. 2001.

[58] A. Basarab, P. Gueth, H. Liebgott, and P. Delachartre, "Phase-based block matching applied to motion estimation with unconventional beamforming strategies," IEEE Trans. Ultrason., Ferroelectr. Freq. Control, vol. 56, no. 5, pp. 945-957, May 2009.

[59] J. Jensen and N. Svendsen, "Calculation of pressure fields from arbitrarily shaped, apodized, and excited ultrasound transducers," IEEE Trans. Ultrason., Ferroelectr. Freq. Control, vol. 39, no. 2, pp. 262-267, Mar. 1992.

[60] J. A. Jensen, "Field: A program for simulating ultrasound systems," in Proc. 10th Nordic-Baltic Conf. Biomed. Imag., 1996, vol. 34, pp. 351-353.

[61] T. Dietenbeck, M. Alessandrini, D. Barbosa, J. D’Hooge, D. Friboulet, and O. Bernard, "Detection of the whole myocardium in 2d-echocardiography for multiple orientations using a geometrically constrained level-set," Med. Image Anal., vol. 16, no. 2, pp. 386-401, 2012.
[62] M. Otte and H. Nagel, "Optical flow estimation: Advances and comparisons," in Computer Vision ECCV'94, J.-O. Eklundh, Ed. New York: Springer, 1994, vol. 800, Lecture Notes in Computer Science, pp. 49-60.

[63] B. Heyde, R. Jasaityte, D. Barbosa, V. Robesyn, S. Bouchez, P. Wouters, F. Maes, P. Claus, and J. D'Hooge, "Elastic image registration versus speckle tracking for 2-d myocardial motion estimation: A direct comparison in vivo," IEEE Trans. Med. Imag., vol. 32, no. 2, pp. 449-459, Jan. 2013.

[64] M. D. Cerqueira, N. J. Weissman, V. Dilsizian, A. K. Jacobs, S. Kaul, W. K. Laskey, D. J. Pennell, J. A. Rumberger, T. Ryan, and M. S. Verani, "Standardized myocardial segmentation and nomenclature for tomographic imaging of the heart: A statement for healthcare professionals from the Cardiac Imaging Committee of the Council on Clinical Cardiology of the American Heart Association," Circulation vol. 105, no. 4, pp. 539-542, 2002.

[65] W. Daniel, Applied Nonparametric Statistics. Pacific Grove, CA: Duxbury Thomson Learn., 2000.

[66] V. Chalana and Y. Kim, "A methodology for evaluation of boundary detection algorithms on medical images," IEEE Trans. Med. Imag., vol. 16, no. 5, pp. 642-652, Oct. 1997.

[67] D. Liu and E. Ebbini, "Real-time 2-d temperature imaging using ultrasound," IEEE Trans. Biomed. Eng., vol. 57, no. 1, pp. 12-16, Jan. 2010 .

[68] T. Yingchoncharoen, S. Agarwal, Z. B. Popovi, and T. H. Marwick, "Normal ranges of left ventricular strain: A meta-analysis," J. Am. Soc. Echocardiogr., vol. 26, no. 2, pp. 185-191, 2013.

[69] B. Andres, F. Hamprecht, and C. Garbe, "Selection of local optical flow models by means of residual analysis," in Pattern Recognition. New York: Springer, 2007, vol. 4713, Lecture Notes in Computer Science, pp. 72-81.

[70] M. Pihl and J. Jensen, "Measuring 3D velocity vectors using the transverse oscillation method," in Proc. 2012 IEEE Int. Ultrason. Symp., Oct. 2012, pp. 1881-1885.

[71] M. Pihl, M. Stuart, B. Tomov, J. Hansen, M. Rasmussen, and J. Jensen, "Preliminary examples of 3D vector flow imaging," in Proc. SPIE Med. Imag. Conf., 2013, p. $86750 \mathrm{H}$.

[72] S. Salles, H. Liebgott, D. Garcia, and D. Vray, "Real time 3D US tagging combined with 3D phase-based motion estimation," in Proc. IEEE Int. Ultrason. Symp., 2013, pp. 585-588. 